\title{
Multi-dimensional, fully-implicit, spectral method for the Vlasov-Maxwell equations with exact conservation laws in discrete form
}

\author{
G. L. Delzanno ${ }^{\mathrm{a}}$, \\ a T-5 Applied Mathematics and Plasma Physics Group, Los Alamos National \\ Laboratory, Los Alamos, NM 87545, USA
}

\begin{abstract}
A spectral method for the numerical solution of the multi-dimensional VlasovMaxwell equations is presented. The plasma distribution function is expanded in Fourier (for the spatial part) and Hermite (for the velocity part) basis functions, leading to a truncated system of ordinary differential equations for the expansion coefficients (moments) that is discretized with an implicit, second order accurate Crank-Nicolson time discretization. The discrete non-linear system is solved with a preconditioned Jacobian-Free Newton-Krylov method.

It is shown analytically that the Fourier-Hermite method features exact conservation laws for total mass, momentum and energy in discrete form. Standard tests involving plasma waves and the whistler instability confirm the validity of the conservation laws numerically. The whistler instability test also shows that we can step over the fastest time scale in the system without incurring in numerical instabilities. Some preconditioning strategies are presented, showing that the number of linear iterations of the Krylov solver can be drastically reduced and a significant gain in performance can be obtained.
\end{abstract}

\section{Introduction}

Collisionless magnetized plasmas are well described by the Vlasov-Maxwell equations [1]. The plasma is represented by a distribution function in six dimensional space, which evolves self-consistently with the electromagnetic field given by Maxwell's equations. Because of the high dimensionality and the large

Email address: delzanno@lanl.gov (G. L. Delzanno). 
separation of scales typical of magnetized plasmas, the Vlasov-Maxwell equations pose a serious challenge to the numerical modeler and their numerical solution has been an active research area for decades.

There are essentially three approaches to the numerical solution of the VlasovMaxwell equations. One is the Particle-In-Cell (PIC) approach [2], where phase space is discretized by introducing a number of macroparticles and following their characteristics. A second approach is called Eulerian Vlasov [3-5], where phase space is discretized by introducing a computational grid in both spatial and velocity coordinates. A third approach is spectral (also referred to as transform methods) [6-11]. Here the velocity part of the distribution function is expanded in basis functions (typically Fourier or Hermite) to obtain a truncated system of moment equations. For each of these methods, a further important classification involves the time discretization. Explicit methods are typically easy to implement but suffer from stringent numerical stability constraints that compromise their applicability to multiscale problems. Semiimplicit methods have a much larger stability domain than explicit methods, but large numerical error can accumulate over time (with the numerical solution drifting away from the actual solution) and lack conservation laws in the discrete. Fully implicit methods, on the other hand, have the largest stability domain and can satisfy exact conservation laws in the discrete, but are harder to implement.

PIC (mostly explicit) is by far the most widely used method in the community. This is due to its relative simplicity and to the fact that in the early days, where computational resources were limited, PIC investigations were successful with a relatively low number of macroparticles. However, a major and well recognized problem of PIC is its intrinsic statistical noise. On the contrary, Eulerian Vlasov and spectral methods do not suffer from statistical noise and can therefore (at least in principle) explore regimes that are hard for PIC. Their main limitation so far has been the need for large computational resources due to the high dimensionality. For spectral methods, this is brought by the fact that one might need a large number of moments to capture the details of the distribution function. However, the continuous advance in computer power mitigates these limitations and continues to drive research in Eulerian Vlasov [12-17] and spectral methods [18-22].

Among the various possible algorithmic choices available in the literature (across or within each of the methods discussed above), a natural guiding principle is to favor numerical schemes that feature conservation properties (for instance for mass, momentum and energy). This has been a rather difficult task in PIC. The so-called explicit momentum conserving PIC [2] only conserves mass and momentum on a uniform computational grid. The explicit energy-conserving PIC [23] conserves only mass and energy, the latter in the limit of vanishing time step. A recent variational formulation [24] achieves 
momentum and energy conservation, again in the limit of small time step. Recent algorithmic advances enabled by fully implicit time stepping have led to a true charge- and energy-conserving PIC [25,26] (i.e. with total energy conserved in a finite time step) but still lack momentum conservation. Indeed, in PIC it does not appear possible to conserve momentum and energy simultaneously for finite time step [2]. On the other hand recent work has shown that this is possible for spectral methods due to implicit time stepping [22]. In Ref. [22] exact mass, momentum and energy conservation was achieved for the first time in one dimension and in the electrostatic limit (i.e. for the Vlasov-Poisson system) with finite time step (for reference, the numerical schemes discussed in Refs. $[10,11]$ achieved exact conservation laws in the limit of vanishing time step). Some important progress in enforcing exact discrete conservation laws has also been achieved for Eulerian Vlasov methods [27-29].

In this paper we focus on spectral methods mainly for two reasons. First, these are the only methods that bridge naturally between a fluid/macroscopic (i.e. with a low number of moments) and a kinetic/microscopic (with a large number of moments) description of the plasma [30], and therefore offer the potential to optimize/minimize the number of degrees of freedom that are necessary to achieve a given resolution in phase space. PIC and Eulerian Vlasov codes cannot do this, as they resolve the full distribution function everywhere in the computational domain. Second, under certain conditions, spectral methods can have exact conservation properties. Indeed, the main contribution of the present paper is the development of a multi-dimensional, electromagnetic, fully implicit spectral method. The fully implicit time discretization enables a large stability domain (relative to its explicit and semi-implicit equivalent) and the exact conservation of mass, momentum and energy exactly (for spatially periodic boundary conditions). To the best of our knowledge, this is done for the first time in the context of spectral methods for the Vlasov-Maxwell equations. We note, however, that spectral methods have also some important drawbacks that will need to be addressed to make such methods more competitive. Some authors [31] have reported a slow convergence of the Hermite expansion in velocity space (although it is worth mentioning that the same reference also successfully applies a method to accelerate the convergence of the expansion) and one could expect the method to be inefficient when strong nonMaxwellian features develop. The optimization of the basis function (including the possibility of shifting and/or splitting it in multiple distributions) is at this point an open but crucial problem. Furthermore, the approach lacks a maximum principle and consequently positivity is not enforced. This aspect can be mitigated by introducing an artificial collisional operator to damp the (unavoidable) phase space filamentation typical of collisionless plasmas and by using more modes in the expansion. 
The paper is organized as follows. In Sec. 2 we introduce the Vlasov-Maxwell equations and the expansion of the spatial (velocity) part of the distribution in Fourier (Hermite) modes. The resulting set of ordinary differential equations for the moments of the expansion is discretized implicitly with a second order accurate Crank-Nicolson scheme. In Sec. 3 we prove analytically that this numerical scheme conserves mass, momentum and energy exactly. In Sec. 4 we study the numerical (linear) stability of the Crank-Nicolson discretization scheme. In Sec. 5 we discuss details of the numerical implementation, with emphasis on the preconditioned Jacobian-Free Newton-Krylov technique used to solve the non-linear equations. In Sec. 6 we present some standard test of the numerical code (involving plasma waves and the whistler instability) proving the validity of the conservation laws numerically. The whistler instability test also shows that we can step over the electron plasma frequency (the fastest frequency in the system for the parameters chosen) without incurring in numerical instabilities, and proves the effectiveness of the preconditioning techniques discussed in Sec. 5. We draw conclusions in Sec. 7.

\section{The method}

We study the dynamics of a collisionless, magnetized plasma consisting of electrons and singly charged ions evolving according to the Vlasov equation. We introduce the following normalization: time is normalized to the electron plasma frequency $\omega_{p e}=\sqrt{\frac{e^{2} n_{0}}{\varepsilon_{0} m_{e}}}$ (where $e$ is the elementary charge, $m_{e}$ is the electron mass, $\varepsilon_{0}$ is vacuum permittivity, and $n_{0}$ is a reference density), velocity to the speed of light $c$, the spatial coordinate to the electron inertial length $d_{e}=c / \omega_{p e}$, the magnetic field to a reference magnetic field $B_{0}$, and the electric field to $c B_{0}$. The dimensionless Vlasov equation for the distribution function $f_{s}$ of each plasma species reads:

$$
\frac{\partial f_{s}}{\partial t}+\mathbf{v} \cdot \nabla_{x} f_{s}+\frac{q_{s}}{e} \frac{\omega_{c s}}{\omega_{p e}}(\mathbf{E}+\mathbf{v} \times \mathbf{B}) \cdot \nabla_{v} f_{s}=0
$$

where subscript $s=e,(i)$ labels electrons (ions), $q_{s}$ is the particle charge $\left(q_{s}= \pm e\right), m_{s}$ is the particle mass, and $\mathbf{E}(\mathbf{B})$ is the electric (magnetic) field. The cyclotron frequency is defined as $\omega_{c s}=e B_{0} / m_{s}$. The plasma distribution function depends on three spatial coordinates, three velocity coordinates, and time, and $\nabla_{x(v)}$ represents the gradient operator in the relevant space. Equation (1) is coupled to Maxwell's equations via the external and plasma-induced electromagnetic field: 


$$
\begin{aligned}
& \frac{\partial \mathbf{B}}{\partial t}=-\nabla \times \mathbf{E} \\
& \frac{\partial \mathbf{E}}{\partial t}=\nabla \times \mathbf{B}-\frac{\omega_{p e}}{\omega_{c e}}\left(\int_{-\infty}^{+\infty} \mathbf{v} f_{i} d \mathbf{v}-\int_{-\infty}^{+\infty} \mathbf{v} f_{e} d \mathbf{v}\right) \\
& \nabla \cdot \mathbf{E}=\frac{\omega_{p e}}{\omega_{c e}}\left(\int_{-\infty}^{+\infty} f_{i} d \mathbf{v}-\int_{-\infty}^{+\infty} f_{e} d \mathbf{v}\right) \\
& \nabla \cdot \mathbf{B}=0
\end{aligned}
$$

We employ an expansion in Hermite polynomials for the velocity space part of the plasma distribution function. We introduce the following asymmetric basis [10]

$$
\begin{aligned}
& \Psi_{n}\left(\xi_{\beta}^{s}\right)=\left(\pi 2^{n} n !\right)^{-1 / 2} H_{n}\left(\xi_{\beta}^{s}\right) \exp \left[-\left(\xi_{\beta}^{s}\right)^{2}\right], \\
& \Psi^{n}\left(\xi_{\beta}^{s}\right)=\left(2^{n} n !\right)^{-1 / 2} H_{n}\left(\xi_{\beta}^{s}\right),
\end{aligned}
$$

where $\xi_{\beta}^{s}=\left(v_{\beta}-u_{\beta}^{s}\right) / \alpha_{\beta}^{s}\left(\beta=x, y, z\right.$ and $u_{\beta}^{s}$ and $\alpha_{\beta}^{s}$ are constants that depend on the plasma species) and $H_{n}$ is the n-th Hermite polynomial. The plasma distribution function is defined by a truncated expansion

$$
f_{\alpha}(\mathbf{x}, \mathbf{v}, t)=\sum_{n=0}^{N_{n}-1} \sum_{m=0}^{N_{m}-1} \sum_{p=0}^{N_{p}-1} C_{n, m, p}^{s}(\mathbf{x}, t) \Psi_{n}\left(\xi_{x}^{s}\right) \Psi_{m}\left(\xi_{y}^{s}\right) \Psi_{p}\left(\xi_{z}^{s}\right)
$$

where $N_{m(m, p)}$ is the total number of polynomials in the $v_{x}\left(v_{y}, v_{z}\right)$ direction. Multiplying Eq. (1) by $\Psi^{\alpha}\left(\xi_{x}^{s}\right) \Psi^{\beta}\left(\xi_{y}^{s}\right) \Psi^{\gamma}\left(\xi_{z}^{s}\right)$, integrating over $d \xi_{x}^{s} d \xi_{y}^{s} d \xi_{z}^{s}$, and using the orthogonality relations of the Hermite polynomials [32], Eq. (1) transforms into a set of partial differential equations for the coefficients of the expansion: 


$$
\begin{aligned}
& \frac{\partial C_{n, m, p}^{s}}{\partial t}=-\alpha_{x}^{s}\left(\sqrt{\frac{n+1}{2}} \frac{\partial C_{n+1, m, p}^{s}}{\partial x}+\sqrt{\frac{n}{2}} \frac{\partial C_{n-1, m, p}^{s}}{\partial x}+\sqrt{\frac{u_{x}^{s}}{\alpha_{x}^{s}}} \frac{\partial C_{n, m, p}^{s}}{\partial x}\right)- \\
& \alpha_{y}^{s}\left(\sqrt{\frac{m+1}{2}} \frac{\partial C_{n, m+1, p}^{s}}{\partial y}+\sqrt{\frac{m}{2}} \frac{\partial C_{n, m-1, p}^{s}}{\partial y}+\sqrt{\frac{u_{y}^{s}}{\alpha_{y}^{s}}} \frac{\partial C_{n, m, p}^{s}}{\partial y}\right)- \\
& \alpha_{z}^{s}\left(\sqrt{\frac{p+1}{2}} \frac{\partial C_{n, m, p+1}^{s}}{\partial z}+\sqrt{\frac{p}{2}} \frac{\partial C_{n, m, p-1}^{s}}{\partial z}+\sqrt{\frac{u_{z}^{s}}{\alpha_{z}^{s}}} \frac{\partial C_{n, m, p}^{s}}{\partial z}\right)+ \\
& \frac{q_{s}}{e} \frac{\omega_{c s}}{\omega_{p e}}\left(\frac{\sqrt{2 n}}{\alpha_{x}^{s}} E_{x} C_{n-1, m, p}+\frac{\sqrt{2 m}}{\alpha_{y}^{s}} E_{y} C_{n, m-1, p}+\frac{\sqrt{2 p}}{\alpha_{z}^{s}} E_{z} C_{n, m, p-1}\right)+ \\
& \frac{q_{s}}{e} \frac{\omega_{c s}}{\omega_{p e}} B_{x}\left[\sqrt{m p}\left(\frac{\alpha_{z}^{s}}{\alpha_{y}^{s}}-\frac{\alpha_{y}^{s}}{\alpha_{z}^{s}}\right) C_{n, m-1, p-1}^{s}+\sqrt{m(p+1)} \frac{\alpha_{z}^{s}}{\alpha_{y}^{s}} C_{n, m-1, p+1}^{s}-\sqrt{(m+1) p} \frac{\alpha_{y}^{s}}{\alpha_{z}^{s}} C_{n, m+1, p-1}^{s}+\right. \\
& \left.\sqrt{2 m} \frac{u_{z}^{s}}{\alpha_{y}^{s}} C_{n, m-1, p}^{s}-\sqrt{2 p} \frac{u_{y}^{s}}{\alpha_{z}^{s}} C_{n, m, p-1}^{s}\right]+ \\
& \frac{q_{s}}{e} \frac{\omega_{c s}}{\omega_{p e}} B_{y}\left[\sqrt{n p}\left(\frac{\alpha_{x}^{s}}{\alpha_{z}^{s}}-\frac{\alpha_{z}^{s}}{\alpha_{x}^{s}}\right) C_{n-1, m, p-1}^{s}+\sqrt{(n+1) p} \frac{\alpha_{x}^{s}}{\alpha_{z}^{s}} C_{n+1, m, p-1}^{s}-\sqrt{n(p+1)} \frac{\alpha_{z}^{s}}{\alpha_{x}^{s}} C_{n-1, m, p+1}^{s}+\right. \\
& \left.\sqrt{2 p} \frac{u_{x}^{s}}{\alpha_{z}^{s}} C_{n, m, p-1}^{s}-\sqrt{2 n} \frac{u_{z}^{s}}{\alpha_{x}^{s}} C_{n-1, m, p}^{s}\right]+ \\
& \frac{q_{s}}{e} \frac{\omega_{c s}}{\omega_{p e}} B_{z}\left[\sqrt{n m}\left(\frac{\alpha_{y}^{s}}{\alpha_{x}^{s}}-\frac{\alpha_{x}^{s}}{\alpha_{y}^{s}}\right) C_{n-1, m-1, p}^{s}+\sqrt{n(m+1)} \frac{\alpha_{y}^{s}}{\alpha_{x}^{s}} C_{n-1, m+1, p}^{s}-\sqrt{(n+1) m} \frac{\alpha_{x}^{s}}{\alpha_{y}^{s}} C_{n+1, m-1, p}^{s}+\right. \\
& \left.\sqrt{2 n} \frac{u_{y}^{s}}{\alpha_{x}^{s}} C_{n-1, m, p}^{s}-\sqrt{2 m} \frac{u_{x}^{s}}{\alpha_{y}^{s}} C_{n, m-1, p}^{s}\right] .
\end{aligned}
$$

In deriving Eq. (9), the following properties of the Hermite basis [32] have been used

$$
\begin{aligned}
& v_{\beta} \Psi_{n}\left(\xi_{\beta}^{s}\right)=\alpha_{\beta}^{s} \sqrt{\frac{n+1}{2}} \Psi_{n+1}\left(\xi_{\beta}^{s}\right)+\alpha_{\beta}^{s} \sqrt{\frac{n}{2}} \Psi_{n-1}\left(\xi_{\beta}^{s}\right)+u_{\beta}^{s} \Psi_{n}\left(\xi_{\beta}^{s}\right) \\
& \frac{d \Psi_{n}\left(\xi_{\beta}^{s}\right)}{d v_{\beta}}=-\frac{\sqrt{2(n+1)}}{\alpha_{\beta}^{s}} \Psi_{n+1}\left(\xi_{\beta}^{s}\right) \\
& \int_{-\infty}^{+\infty} \Psi_{n}\left(\xi_{\beta}^{s}\right) \Psi^{m}\left(\xi_{\beta}^{s}\right) d \xi_{\beta}^{s}=\delta_{n, m},
\end{aligned}
$$

with $\delta_{n, m}$ the Kronecker delta. Note that Eq. (9) for $C_{n, m, p}^{s}$ requires knowledge of higher order moments, for instance $C_{n+1, m, p}^{s}$. The system is truncated by imposing that

$$
C_{n, m, p}^{s}=0, \quad \text { for } n \geq N_{n}, m \geq N_{m}, p \geq N_{p} .
$$




\subsection{Spatial discretization}

In what follows, we consider a Cartesian domain of size $\left[0, L_{x}\right] \times\left[0, L_{y}\right] \times\left[0, L_{z}\right]$ and assume periodic boundary conditions for the spatial coordinates.

We expand the spatial part of the distribution function in Fourier modes

$$
C_{n, m, p}^{s}(\mathbf{x}, t)=\sum_{k_{x}=-N_{x} / 2}^{N_{x} / 2} \sum_{k_{y}=-N_{y} / 2}^{N_{y} / 2} \sum_{k_{z}=-N_{z} / 2}^{N_{z} / 2} C_{n, m, p}^{k_{x}, k_{y}, k_{z}, s}(t) \exp \left[2 \pi i\left(\frac{k_{x} x}{L_{x}}+\frac{k_{y} y}{L_{y}}+\frac{k_{z} z}{L_{z}}\right)\right]
$$

and similarly for the electromagnetic field. In Eq. (14), the total number of modes in the $x(y, z)$ direction is $N_{x}+1\left(N_{y}+1, N_{z}+1\right)$. We note that the Fourier global basis is particularly suited for wave turbulence problems. Other approaches featuring a local basis (via a Galerkin expansion) will be explored in the future.

Multiplying Eq. (9) by $\exp \left[-2 \pi i\left(\frac{\alpha x}{L_{x}}+\frac{\beta y}{L_{y}}+\frac{\gamma z}{L_{z}}\right)\right]$, integrating over $d x d y d z$ and using the orthogonality of the Fourier basis, Eq. (9) transforms into a set of ordinary differential equations 


$$
\begin{aligned}
& \frac{d C_{n, m_{y}, p}^{k_{x}, k_{y}, k_{z}, s}}{d t}=-\frac{2 \pi i k_{x}}{L_{x}} \alpha_{x}^{s}\left(\sqrt{\frac{n+1}{2}} C_{n+1, m, p}^{k_{x}, k_{y}, k_{z}, s}+\sqrt{\frac{n}{2}} C_{n-1, m, p}^{k_{x}, k_{y}, k_{z}, s}+\sqrt{\frac{u_{x}^{s}}{\alpha_{x}^{s}}} C_{n, m, p}^{k_{x}, k_{y}, k_{z}, s}\right)- \\
& \frac{2 \pi i k_{y}}{L_{y}} \alpha_{y}^{s}\left(\sqrt{\frac{m+1}{2}} C_{n, m+1, p}^{k_{x}, k_{y}, k_{z}, s}+\sqrt{\frac{m}{2}} C_{n, m-1, p}^{k_{x}, k_{y}, k_{z}, s}+\sqrt{\frac{u_{y}^{s}}{\alpha_{y}^{s}}} C_{n, m, p}^{k_{x}, k_{y}, k_{z}, s}\right)- \\
& \frac{2 \pi i k_{z}}{L_{z}} \alpha_{z}^{s}\left(\sqrt{\frac{p+1}{2}} C_{n, m, p+1}^{k_{x}, k_{y}, k_{z}, s}+\sqrt{\frac{p}{2}} C_{n, m, p-1}^{k_{x}, k_{y}, k_{z}, s}+\sqrt{\frac{u_{z}^{s}}{\alpha_{z}^{s}}} C_{n, m, p}^{k_{x}, k_{y}, k_{z}, s}\right)+ \\
& \frac{q_{s}}{e} \frac{\omega_{c s}}{\omega_{p e}}\left\{\frac{\sqrt{2 n}}{\alpha_{x}^{s}}\left[E_{x} * C_{n-1, m, p}\right]_{k_{x}, k_{y}, k_{z}}+\frac{\sqrt{2 m}}{\alpha_{y}^{s}}\left[E_{y} * C_{n, m-1, p}\right]_{k_{x}, k_{y}, k_{z}}+\frac{\sqrt{2 p}}{\alpha_{z}^{s}}\left[E_{z} * C_{n, m, p-1}\right]_{k_{x}, k_{y}, k_{z}}\right\}+ \\
& \frac{q_{s}}{e} \frac{\omega_{c s}}{\omega_{p e}}\left\{\sqrt{m p}\left(\frac{\alpha_{z}^{s}}{\alpha_{y}^{s}}-\frac{\alpha_{y}^{s}}{\alpha_{z}^{s}}\right)\left[B_{x} * C_{n, m-1, p-1}^{s}\right]_{k_{x}, k_{y}, k_{z}}+\sqrt{m(p+1)} \frac{\alpha_{z}^{s}}{\alpha_{y}^{s}}\left[B_{x} * C_{n, m-1, p+1}^{s}\right]_{k_{x}, k_{y}, k_{z}}-\right. \\
& \sqrt{(m+1) p} \frac{\alpha_{y}^{s}}{\alpha_{z}^{s}}\left[B_{x} * C_{n, m+1, p-1}^{s}\right]_{k_{x}, k_{y}, k_{z}}+\sqrt{2 m} \frac{u_{z}^{s}}{\alpha_{y}^{s}}\left[B_{x} * C_{n, m-1, p}^{s}\right]_{k_{x}, k_{y}, k_{z}}- \\
& \left.\sqrt{2 p} \frac{u_{y}^{s}}{\alpha_{z}^{s}}\left[B_{x} * C_{n, m, p-1}^{s}\right]_{k_{x}, k_{y}, k_{z}}\right\}+ \\
& \frac{q_{s}}{e} \frac{\omega_{c s}}{\omega_{p e}}\left\{\sqrt{n p}\left(\frac{\alpha_{x}^{s}}{\alpha_{z}^{s}}-\frac{\alpha_{z}^{s}}{\alpha_{x}^{s}}\right)\left[B_{y} * C_{n-1, m, p-1}^{s}\right]_{k_{x}, k_{y}, k_{z}}+\sqrt{(n+1) p} \frac{\alpha_{x}^{s}}{\alpha_{z}^{s}}\left[B_{y} * C_{n+1, m, p-1}^{s}\right]_{k_{x}, k_{y}, k_{z}}-\right. \\
& \sqrt{n(p+1)} \frac{\alpha_{z}^{s}}{\alpha_{x}^{s}}\left[B_{y} * C_{n-1, m, p+1}^{s}\right]_{k_{x}, k_{y}, k_{z}}+\sqrt{2 p} \frac{u_{x}^{s}}{\alpha_{z}^{s}}\left[B_{y} * C_{n, m, p-1}^{s}\right]_{k_{x}, k_{y}, k_{z}}- \\
& \left.\sqrt{2 n} \frac{u_{z}^{s}}{\alpha_{x}^{s}}\left[B_{y} * C_{n-1, m, p}^{s}\right]_{k_{x}, k_{y}, k_{z}}\right\}+ \\
& \frac{q_{s}}{e} \frac{\omega_{c s}}{\omega_{p e}}\left\{\sqrt{n m}\left(\frac{\alpha_{y}^{s}}{\alpha_{x}^{s}}-\frac{\alpha_{x}^{s}}{\alpha_{y}^{s}}\right)\left[B_{z} * C_{n-1, m-1, p}^{s}\right]_{k_{x}, k_{y}, k_{z}}+\sqrt{n(m+1)} \frac{\alpha_{y}^{s}}{\alpha_{x}^{s}}\left[B_{z} * C_{n-1, m+1, p}^{s}\right]_{k_{x}, k_{y}, k_{z}}-\right. \\
& \sqrt{(n+1) m} \frac{\alpha_{x}^{s}}{\alpha_{y}^{s}}\left[B_{z} * C_{n+1, m-1, p}^{s}\right]_{k_{x}, k_{y}, k_{z}}+\sqrt{2 n} \frac{u_{y}^{s}}{\alpha_{x}^{s}}\left[B_{z} * C_{n-1, m, p}^{s}\right]_{k_{x}, k_{y}, k_{z}}- \\
& \left.\sqrt{2 m} \frac{u_{x}^{s}}{\alpha_{y}^{s}}\left[B_{z} * C_{n, m-1, p}^{s}\right]_{k_{x}, k_{y}, k_{z}}\right\}
\end{aligned}
$$

where the nonlinearities of Eq. (9) in physical space lead to convolutions in Fourier space defined as

$$
\left[H * C_{n, m, p}\right]_{k_{x}, k_{y}, k_{z}}=\sum_{k_{x}^{\prime}=-N_{x} / 2}^{N_{x} / 2} \sum_{k_{y}^{\prime}=-N_{y} / 2}^{N_{y} / 2} \sum_{k_{z}^{\prime}=-N_{z} / 2}^{N_{z} / 2} H^{k_{x}-k_{x}^{\prime}, k_{y}-k_{y}^{\prime}, k_{z}-k_{z}^{\prime}} C_{n, m, p}^{k_{x}^{\prime}, k_{y}^{\prime}, k_{z}^{\prime}, s}
$$

( $H$ is an arbitrary function). Similarly, Maxwell's equations in Fourier space become 


$$
\begin{aligned}
& \frac{d B_{x}^{k_{x}, k_{y}, k_{z}}}{d t}=-2 \pi i\left(\frac{k_{y}}{L_{y}} E_{z}^{k_{x}, k_{y}, k_{z}}-\frac{k_{z}}{L_{z}} E_{y}^{k_{x}, k_{y}, k_{z}}\right), \\
& \frac{d B_{y}^{k_{x}, k_{y}, k_{z}}}{d t}=-2 \pi i\left(\frac{k_{z}}{L_{z}} E_{x}^{k_{x}, k_{y}, k_{z}}-\frac{k_{x}}{L_{x}} E_{z}^{k_{x}, k_{y}, k_{z}}\right), \\
& \frac{d B_{z}^{k_{x}, k_{y}, k_{z}}}{d t}=-2 \pi i\left(\frac{k_{x}}{L_{x}} E_{y}^{k_{x}, k_{y}, k_{z}}-\frac{k_{y}}{L_{y}} E_{x}^{k_{x}, k_{y}, k_{z}}\right), \\
& \frac{d E_{x}^{k_{x}, k_{y}, k_{z}}}{d t}=2 \pi i\left(\frac{k_{y}}{L_{y}} B_{z}^{k_{x}, k_{y}, k_{z}}-\frac{k_{z}}{L_{z}} B_{y}^{k_{x}, k_{y}, k_{z}}\right)- \\
& \frac{\omega_{p e}}{\omega_{c e}} \sum_{s=e, i} \frac{q_{s}}{e}\left[\alpha_{x}^{s} \alpha_{y}^{s} \alpha_{z}^{s}\left(\frac{\alpha_{x}^{s}}{\sqrt{2}} C_{1,0,0}^{k_{x}, k_{y}, k_{z}, s}+u_{x}^{s} C_{0,0,0}^{k_{x}, k_{y}, k_{z}, s}\right)\right], \\
& \frac{d E_{y}^{k_{x}, k_{y}, k_{z}}}{d t}=2 \pi i\left(\frac{k_{z}}{L_{z}} B_{x}^{k_{x}, k_{y}, k_{z}}-\frac{k_{x}}{L_{x}} B_{z}^{k_{x}, k_{y}, k_{z}}\right)- \\
& \frac{\omega_{p e}}{\omega_{c e}} \sum_{s=e, i} \frac{q_{s}}{e}\left[\alpha_{x}^{s} \alpha_{y}^{s} \alpha_{z}^{s}\left(\frac{\alpha_{y}^{s}}{\sqrt{2}} C_{0,1,0}^{k_{x}, k_{y}, k_{z}, s}+u_{y}^{s} C_{0,0,0}^{k_{x}, k_{y}, k_{z}, s}\right)\right], \\
& \frac{\omega_{p e}, k_{y}, k_{z}}{\omega_{c e}} \sum_{s=e, i} \frac{q_{s}}{e}\left[\alpha_{x}^{s} \alpha_{y}^{s} \alpha_{z}^{s}\left(\frac{\alpha_{z}^{s}}{\sqrt{2}} C_{0,0,1}^{k_{x}, k_{y}, k_{z}, s}+u_{z}^{s} C_{0,0,0}^{k_{x}, k_{y}, k_{z}, s}\right)\right] .
\end{aligned}
$$

It can be easily verified that the Fourier representation of Faraday's and Ampere's laws [Eqs. (2) and (3)] preserve the constraints (4) and (5). In other words, if these constraints are satisfied at time $t=0$, then they are satisfied at all times.

In vectorial form, Eqs. (15) and (17) become

$$
\begin{aligned}
& \frac{d \mathbf{C}}{d t}=\mathbb{L}_{1} \mathbf{C}+\mathcal{N}(\mathbf{C}, \mathbf{F}), \\
& \frac{d \mathbf{F}}{d t}=\mathbb{L}_{2} \mathbf{C}+\mathbb{L}_{3} \mathbf{F}
\end{aligned}
$$

where

$$
\mathbf{C}=\left[\begin{array}{l}
\mathbf{C}^{e} \\
\mathbf{C}^{i}
\end{array}\right]
$$

and 


$$
\mathbf{C}^{s}=\left[\begin{array}{c}
\mathbf{C}_{0,0,0}^{s} \\
\mathbf{C}_{1,0,0}^{s} \\
\cdots \\
\mathbf{C}_{N_{n}, 0,0}^{s} \\
\mathbf{C}_{0,1,0}^{s} \\
\ldots \\
\mathbf{C}_{0, N_{m}, 0}^{s} \\
\mathbf{C}_{0,0,1}^{s} \\
\cdots \\
\mathbf{C}_{0,0, N_{p}}^{s}
\end{array}\right], \mathbf{F}=\left[\begin{array}{c}
\mathbf{E}_{x} \\
\mathbf{E}_{y} \\
\mathbf{E}_{z} \\
\mathbf{B}_{x} \\
\mathbf{B}_{y} \\
\mathbf{B}_{z}
\end{array}\right] .
$$

Each block in Eqs. (20) is associated with the spatial discretization as, for instance,

$$
\begin{gathered}
\mathbf{C}_{0,0,0}^{s}=\left[C_{0,0,0}^{-N_{x} / 2,-N_{y} / 2,-N_{z} / 2, s}, C_{0,0,0}^{-N_{x} / 2+1,-N_{y} / 2,-N_{z} / 2, s}, \ldots, C_{0,0,0}^{N_{x} / 2,-N_{y} / 2,-N_{z} / 2, s},\right. \\
C_{0,0,0}^{-N_{x} / 2,-N_{y} / 2+1,-N_{z} / 2, s}, \ldots, C_{0,0,0}^{N_{x} / 2,-N_{y} / 2+1,-N_{z} / 2, s}, \ldots, C_{0,0,0}^{-N_{x} / 2, N_{y} / 2,-N_{z} / 2, s}, \\
\quad \ldots, C_{0,0,0}^{N_{x} / 2, N_{y} / 2,-N_{z} / 2, s}, C_{0,0,0}^{-N_{x} / 2,-N_{y} / 2,-N_{z} / 2+1, s}, \ldots, C_{0,0,0}^{N_{x} / 2,-N_{y} / 2,-N_{z} / 2+1, s}, \\
\quad \ldots, C_{0,0,0}^{-N_{x} / 2, N_{y} / 2,-N_{z} / 2+1, s}, \ldots, C_{0,0,0}^{N_{x} / 2, N_{y} / 2,-N_{z} / 2+1, s}, \ldots, C_{0,0,0}^{-N_{x} / 2,-N_{y} / 2, N_{z} / 2, s}, \\
\left.\quad \ldots, C_{0,0,0}^{N_{x} / 2,-N_{y} / 2, N_{z} / 2, s}, \ldots, C_{0,0,0}^{-N_{x} / 2, N_{y} / 2, N_{z} / 2, s}, \ldots, C_{0,0,0}^{N_{x} / 2, N_{y} / 2, N_{z} / 2, s}\right]^{T},
\end{gathered}
$$

and ${ }^{T}$ indicates transposition. Furthermore, the matrix $\mathbb{L}_{1}$ corresponds to the linear advection operator on the right hand side of Eq. (15), while $\mathcal{N}$ is the non-linear operator associated with the convolutions. Finally, the matrices $\mathbb{L}_{2}$ and $\mathbb{L}_{3}$ are defined by the right hand side of Eqs. (17).

\subsection{Temporal discretization}

For the temporal discretization, we follow Ref. [22] and employ a second order accurate Crank-Nicolson discretization scheme [33]. It will be shown in Sec. 3 that this choice is critical to ensure that the numerical scheme satisfies certain conservation laws. We introduce the time step $\Delta t$ and label quantities at time $t^{\theta}=\theta \Delta t$ with superscript $\theta: \mathbf{C}\left(t^{\theta}\right)=\mathbf{C}^{\theta}$ and $\mathbf{F}\left(t^{\theta}\right)=\mathbf{F}^{\theta}$. Furthermore, $\mathbf{C}^{t+1 / 2}=\left(\mathbf{C}^{t+1}+\mathbf{C}^{t}\right) / 2$ and similarly for $\mathbf{F}$. Accordingly, the discretized version of Eqs. (18), expressed in residual form, becomes 


$$
\begin{aligned}
& \mathbf{G}_{1}\left(\mathbf{C}^{\theta+1}, \mathbf{F}^{\theta+1}\right)=\mathbf{C}^{\theta+1}-\mathbf{C}^{\theta}-\Delta t\left[\mathbb{L}_{1} \mathbf{C}^{\theta+1 / 2}+\mathcal{N}\left(\mathbf{C}^{\theta+1 / 2}, \mathbf{F}^{\theta+1 / 2}\right)\right]=0 \\
& \mathbf{G}_{2}\left(\mathbf{C}^{\theta+1}, \mathbf{F}^{\theta+1}\right)=\mathbf{F}^{\theta+1}-\mathbf{F}^{\theta}-\Delta t \mathbb{L}_{2} \mathbf{C}^{\theta+1 / 2}-\Delta t \mathbb{L}_{3} \mathbf{F}^{\theta+1 / 2}=0,
\end{aligned}
$$

or, in more compact form, $\mathbf{G}(\mathbf{X})=0$, with $\mathbf{G}=\left[\begin{array}{l}\mathbf{G}_{1} \\ \mathbf{G}_{2}\end{array}\right]$ and $\mathbf{X}=\left[\begin{array}{l}\mathbf{C}^{\theta+1} \\ \mathbf{F}^{\theta+1}\end{array}\right]$.

\section{Conservation laws}

In this Section, we present the conservations laws for the discretized system of equations (22). We introduce some properties of the convolution operator that are useful in deriving the conservation laws. These are

$$
\left[\left(k_{\beta} A\right) * B\right]_{0,0,0}=\left[A *\left(k_{\beta} B\right)\right]_{0,0,0},
$$

where $\beta=x, y, z$ and $A$ and $B$ are two generic vector quantities, and

$$
\left[\left(k_{\beta} A\right) * A\right]_{0,0,0}=0 .
$$

It is also useful to recall that, by definition,

$$
[A * B]_{0,0,0}=[B * A]_{0,0,0} .
$$

Furthermore, we define

$$
\frac{\Delta A}{\Delta t}=\frac{A^{t+1}-A^{t}}{\Delta t}
$$

and note the following property

$$
\left[\frac{\Delta A}{\Delta t} * B^{t+1 / 2}+A^{t+1 / 2} * \frac{\Delta B}{\Delta t}\right]_{0,0,0}=\frac{\Delta[B * A]_{0,0,0}}{\Delta t} .
$$

Finally, we introduce $\alpha^{s}=\alpha_{x}^{s} \alpha_{y}^{s} \alpha_{z}^{s}$ and $L=L_{x} L_{y} L_{z}$.

\subsection{Conservation of mass}

The total mass per species (normalized to $n_{0} d_{e}^{3}$ ) is

$$
M_{\alpha}=\int d \mathbf{x} \int d \mathbf{v} f_{s}=\alpha_{s} L C_{0,0,0}^{0,0,0, s},
$$

implying that

$$
\frac{\Delta M_{\alpha}}{\Delta t}=L \alpha^{s} \frac{\Delta C_{0,0,0}^{0,0,0, s}}{\Delta t} .
$$


It is easy to see from Eqs. (15) and (22) that $\Delta C_{0,0,0}^{0,0,0, s} / \Delta t=0$ and therefore the total mass in the system is conserved.

\subsection{Conservation of momentum}

The mechanical momentum associated with the plasma is defined by

$$
\begin{aligned}
& \mathbf{P}_{\text {mech }}^{s}=\frac{m_{s}}{m_{e}} \int d \mathbf{x} \int d \mathbf{v} \mathbf{v} f_{s} \\
& =L \alpha^{s}\left[\left(\frac{\alpha_{x}^{s}}{\sqrt{2}} C_{1,0,0}^{0,0,0, s}+u_{x}^{s} C_{0,0,0}^{0,0,0, s}\right) \mathbf{e}_{x}+\left(\frac{\alpha_{y}^{s}}{\sqrt{2}} C_{0,1,0}^{0,0,0, s}+u_{y}^{s} C_{0,0,0}^{0,0,0, s}\right) \mathbf{e}_{y}+\right. \\
& \left.\quad\left(\frac{\alpha_{z}^{s}}{\sqrt{2}} C_{0,0,1}^{0,0,0, s}+u_{z}^{s} C_{0,0,0}^{0,0,0, s}\right) \mathbf{e}_{z}\right]
\end{aligned}
$$

where $\mathbf{e}_{\beta}$ is the unit vector along $\beta$. The momentum associated with the electromagnetic field is

$$
\mathbf{P}_{e m}=\left(\frac{\omega_{c e}}{\omega_{p e}}\right)^{2} \int d \mathbf{x} \mathbf{E} \times \mathbf{B}=L\left(\frac{\omega_{c e}}{\omega_{p e}}\right)^{2} \varepsilon_{i j l}\left[E_{i} * B_{j}\right]_{0,0,0} \mathbf{e}_{l},
$$

where $\varepsilon_{i j l}$ is the Levi-Civita tensor. Here and everywhere else in the text summation over repeated indices is implied. Both $\mathbf{P}_{\text {mech }}^{s}$ and $\mathbf{P}_{e m}$ are normalized to $m_{e} c n_{0} d_{e}^{3}$.

We now proceed to prove the conservation of momentum in the $x$ direction. The same proof follows step by step for the other two directions. By taking the variation of $P_{m e c h, x}^{s}$ in a time step $\Delta t$, it follows that

$$
\frac{\Delta P_{m e c h, x}^{s}}{\Delta t}=L \alpha^{s}\left(\frac{\alpha_{x}^{s}}{\sqrt{2}} \frac{\Delta C_{1,0,0}^{0,0,0, s}}{\Delta t}+u_{x}^{s} \frac{\Delta C_{0,0,0}^{0,0,0, s}}{\Delta t}\right) .
$$

In Eq. (32), $\Delta C_{0,0,0}^{0,0,0, s} / \Delta t=0$ because of mass conservation. Using Eqs. (15) and (22) for $C_{1,0,0}^{0,0,0, s}$, one obtains

$$
\begin{gathered}
\frac{\Delta P_{\text {mech }, x}^{s}}{\Delta t}=L \frac{\omega_{c e}}{\omega_{p e}} \alpha^{s} \frac{q_{s}}{e}\left\{\left[E_{x}^{t+1 / 2} * C_{0,0,0}^{s, t+1 / 2}-B_{y}^{t+1 / 2} *\left(\frac{\alpha_{z}^{s}}{\sqrt{2}} C_{0,0,1}^{s, t+1 / 2}+u_{z}^{s} C_{0,0,0}^{s, t+1 / 2}\right)+\right.\right. \\
\left.\left.B_{z}^{t+1 / 2} *\left(\frac{\alpha_{y}^{s}}{\sqrt{2}} C_{0,1,0}^{s, t+1 / 2}+u_{y}^{s} C_{0,0,0}^{s, t+1 / 2}\right)\right]_{0,0,0}\right\} .
\end{gathered}
$$

By using Poisson's equation (4) and Faraday's law (2), the total mechanical momentum can be expressed as 


$$
\begin{aligned}
& \frac{\Delta P_{\text {mech }, x}}{\Delta t}=\sum_{s} \frac{\Delta P_{m e c h, x}^{s}}{\Delta t}=2 \pi i L\left(\frac{\omega_{c e}}{\omega_{p e}}\right)^{2}\left[E_{x}^{t+1 / 2} *\left(\frac{k_{x}}{L_{x}} E_{y}^{t+1 / 2}+\frac{k_{z}}{L_{z}} E_{z}^{t+1 / 2}\right)\right]_{0,0,0}+ \\
& L\left(\frac{\omega_{c e}}{\omega_{p e}}\right)^{2}\left[B_{y}^{t+1 / 2} * \frac{\Delta E_{z}}{\Delta t}-B_{z}^{t+1 / 2} * \frac{\Delta E_{y}}{\Delta t}\right]_{0,0,0}+ \\
& 2 \pi i L\left(\frac{\omega_{c e}}{\omega_{p e}}\right)^{2}\left[B_{y}^{t+1 / 2} *\left(\frac{k_{y}}{L_{y}} B_{x}^{t+1 / 2}\right)+B_{z}^{t+1 / 2} *\left(\frac{k_{z}}{L_{z}} B_{x}^{t+1 / 2}\right)\right]_{0,0,0} .
\end{aligned}
$$

In deriving Eq. (34) we have used property (24). Note that the last term in the square brackets of Eq. (34) is zero, as it can be verified by applying the $\nabla \cdot \mathbf{B}=0$ constraint,

$$
\frac{k_{x}}{L_{x}} B_{x}^{k_{x}, k_{y}, k_{z}}+\frac{k_{y}}{L_{y}} B_{y}^{k_{x}, k_{y}, k_{z}}+\frac{k_{z}}{L_{z}} B_{z}^{k_{x}, k_{y}, k_{z}}=0
$$

and using again property (24). Furthermore, using Faraday's law to express $\left(\frac{k_{y}}{L_{y}}+\frac{k_{z}}{L_{z}}\right) E_{x}^{t+1 / 2}$, Eq. (34) becomes

$\frac{\Delta P_{m e c h, x}}{\Delta t}=L\left(\frac{\omega_{c e}}{\omega_{p e}}\right)^{2}\left[-\frac{\Delta B_{z}}{\Delta t} * E_{y}^{t+1 / 2}+\frac{\Delta B_{y}}{\Delta t} * E_{z}^{t+1 / 2}+B_{y}^{t+1 / 2} * \frac{\Delta E_{z}}{\Delta t}-B_{z}^{t+1 / 2} * \frac{\Delta E_{y}}{\Delta t}\right]_{0,0,0}$.

Properties (25) and (27) lead to

$$
\frac{\Delta P_{m e c h, x}}{\Delta t}=L\left(\frac{\omega_{c e}}{\omega_{p e}}\right)^{2}\left\{\frac{\Delta\left[E_{z} * B_{y}\right]_{0,0,0}}{\Delta t}-\frac{\Delta\left[E_{y} * B_{z}\right]_{0,0,0}}{\Delta t}\right\}
$$

From the $x$ component of the electromagnetic momentum,

$$
P_{e m, x}=L\left(\frac{\omega_{c e}}{\omega_{p e}}\right)^{2}\left[E_{y} * B_{z}-E_{z} * B_{y}\right]_{0,0,0}
$$

it is easy to see that

$$
\frac{\Delta P_{m e c h, x}}{\Delta t}=-\frac{\Delta P_{e m, x}}{\Delta t}
$$

and, therefore, the total momentum in the system is conserved.

\subsection{Conservation of energy}

The total kinetic energy (normalized to $m_{e} c^{2} n_{o} d_{e}^{3}$ ) in the plasma is given by 


$$
\begin{aligned}
W_{k}^{s}= & \frac{1}{2} \frac{m_{s}}{m_{e}} \int d \mathbf{x} \int d \mathbf{v} v_{i} v_{i} f_{s} \\
= & \frac{L}{2} \frac{m_{s}}{m_{e}} \alpha^{s}\left\{\frac{\left(\alpha_{x}^{s}\right)^{2}}{\sqrt{2}} C_{2,0,0}^{0,0,0, s}+\sqrt{2} u_{x}^{s} \alpha_{x}^{s} C_{1,0,0}^{0,0,0, s}+\left[\frac{\left(\alpha_{x}^{s}\right)^{2}}{2}+\left(u_{x}^{s}\right)^{2}\right] C_{0,0,0}^{0,0,0, s}+\right. \\
& \frac{\left(\alpha_{y}^{s}\right)^{2}}{\sqrt{2}} C_{0,2,0}^{0,0,0, s}+\sqrt{2} u_{y}^{s} \alpha_{y}^{s} C_{0,1,0}^{0,0,0, s}+\left[\frac{\left(\alpha_{y}^{s}\right)^{2}}{2}+\left(u_{y}^{s}\right)^{2}\right] C_{0,0,0}^{0,0,0, s}+ \\
& \left.\frac{\left(\alpha_{z}^{s}\right)^{2}}{\sqrt{2}} C_{0,0,2}^{0,0,0, s}+\sqrt{2} u_{z}^{s} \alpha_{z}^{s} C_{0,0,1}^{0,0,0, s}+\left[\frac{\left(\alpha_{z}^{s}\right)^{2}}{2}+\left(u_{z}^{s}\right)^{2}\right] C_{0,0,0}^{0,0,0, s}\right\} .
\end{aligned}
$$

Its variation in a time step is

$$
\begin{aligned}
& \frac{\Delta W_{k}^{s}}{\Delta t}=\frac{L}{2 \sqrt{2}} \alpha^{s} \frac{m_{s}}{m_{e}}\left[\alpha_{x}^{s}\left(\alpha_{x}^{s} \frac{\Delta C_{2,0,0}^{0,0,0, s}}{\Delta t}+2 u_{x}^{s} \frac{\Delta C_{1,0,0}^{0,0,0, s}}{\Delta t}\right)+\alpha_{y}^{s}\left(\alpha_{y}^{s} \frac{\Delta C_{0,2,0}^{0,0,0, s}}{\Delta t}+2 u_{y}^{s} \frac{\Delta C_{0,1,0}^{0,0,0, s}}{\Delta t}\right)+\right. \\
& \left.\quad \alpha_{z}^{s}\left(\alpha_{z}^{s} \frac{\Delta C_{0,0,2}^{0,0,0, s}}{\Delta t}+2 u_{z}^{s} \frac{\Delta C_{0,0,1}^{0,0,0, s}}{\Delta t}\right)\right] .
\end{aligned}
$$

Using Eqs. (15) and (22), the total change in kinetic energy can be expressed as

$$
\begin{aligned}
& \frac{\Delta W_{k}}{\Delta t}=\sum_{s} \frac{\Delta W_{k}^{s}}{\Delta t}=L \frac{\omega_{c e}}{\omega_{p e}} \sum_{s} \frac{q_{s}}{e} \alpha^{s}\left[E_{x}^{t+1 / 2} *\left(\frac{\alpha_{x}^{s}}{\sqrt{2}} C_{1,0,0}^{s, t+1 / 2}+u_{x}^{s} C_{0,0,0}^{s, t+1 / 2}\right)+\right. \\
& \left.E_{y}^{t+1 / 2} *\left(\frac{\alpha_{y}^{s}}{\sqrt{2}} C_{0,1,0}^{s, t+1 / 2}+u_{y}^{s} C_{0,0,0}^{s, t+1 / 2}\right)+E_{z}^{t+1 / 2} *\left(\frac{\alpha_{z}^{s}}{\sqrt{2}} C_{0,0,1}^{s, t+1 / 2}+u_{z}^{s} C_{0,0,0}^{s, t+1 / 2}\right)\right]
\end{aligned}
$$

where all the terms associated with the magnetic field cancel out.

Let us now consider the electromagnetic energy

$$
W_{e m}=\left(\frac{\omega_{c e}}{\omega_{p e}}\right)^{2} \int d \mathbf{x} \frac{\mathbf{E}^{2}+\mathbf{B}^{2}}{2}=\frac{L}{2}\left(\frac{\omega_{c e}}{\omega_{p e}}\right)^{2}\left\{\left[E_{i} * E_{i}+B_{i} * B_{i}\right]_{0,0,0}\right\}
$$

Its rate of change in a time step is given by

$$
\frac{\Delta W_{e m}}{\Delta t}=L\left(\frac{\omega_{c e}}{\omega_{p e}}\right)^{2}\left\{\left[\frac{\Delta E_{i}}{\Delta t} * E_{i}^{t+1 / 2}+\frac{\Delta B_{i}}{\Delta t} * B_{i}^{t+1 / 2}\right]_{0,0,0}\right\}=W_{E}+W_{B}
$$

where property (27) has been used. Inserting Faraday's and Ampere's laws into Eq. (44), it is easy to obtain 


$$
\begin{aligned}
\frac{\Delta W_{e m}}{\Delta t} & =-L \frac{\omega_{c e}}{\omega_{p e}} \sum_{s} \frac{q_{s}}{e} \alpha^{s}\left\{\left[E_{x}^{t+1 / 2} *\left(\frac{\alpha_{x}^{s}}{\sqrt{2}} C_{1,0,0}^{s, t+1 / 2}+u_{x}^{s} C_{0,0,0}^{s, t+1 / 2}\right)+\right.\right. \\
& \left.\left.E_{y}^{t+1 / 2} *\left(\frac{\alpha_{y}^{s}}{\sqrt{2}} C_{0,1,0}^{s, t+1 / 2}+u_{y}^{s} C_{0,0,0}^{s, t+1 / 2}\right)+E_{z}^{t+1 / 2} *\left(\frac{\alpha_{z}^{s}}{\sqrt{2}} C_{0,0,1}^{s, t+1 / 2}+u_{z}^{s} C_{0,0,0}^{s, t+1 / 2}\right)\right]_{0,0,0}\right\} \\
& =-\frac{\Delta W_{k}}{\Delta t}
\end{aligned}
$$

proving the conservation of the total energy in the system.

In summary, we have proven that the Fourier-Hermite representation of the Vlasov-Maxwell equations for a collisionless plasma with periodic boundary conditions, together with an implicit time discretization based on the CrankNicolson scheme, features conservation laws for the total mass, momentum and energy in the discrete. This is a remarkable property of the numerical scheme, ensuring that the dynamics of the system remains constrained by these conservation laws. For comparison, no existing PIC method (explicit or implicit) can conserve mass, momentum and energy simultaneously in a finite time step, and one must always monitor the quantity that is not conserved to guarantee that artificial sources of momentum or energy do not affect the dynamics.

\section{Linear analysis of numerical stability}

In this section, we perform some studies of numerical stability. For simplicity, we focus on linear stability and linearize the equations relative to an equilibrium where the plasma distribution function is an isotropic Maxwellian and the electromagnetic field is zero $\mathbf{F}_{e q}=0$ (subscript 'eq' will be used to label equilibrium quantities). Moreover we consider only one spatial mode $\left(k_{x}, k_{y}, k_{z}\right)$. We note that the system under consideration is stable. For instance, an initial perturbation in the electric field would (Landau) damp away.

The linearized Eqs. (15) and (17) can be cast as

$$
\begin{aligned}
& \frac{d \delta \mathbf{C}}{d t}=\mathbb{L}_{1} \delta \mathbf{C}+\mathcal{N}\left(\mathbf{C}_{e q}, \delta \mathbf{F}\right), \\
& \frac{d \delta \mathbf{F}}{d t}=\mathbb{L}_{2} \delta \mathbf{C}+\mathbb{L}_{3} \delta \mathbf{F},
\end{aligned}
$$

or, more conveniently, as

$$
\frac{d \delta \mathbf{X}}{d t}=\mathbb{A} \delta \mathbf{X}
$$


where $\mathbb{A}$ is the matrix corresponding to the linearized Eqs. (46), whose dimension is $\left(2 N_{n} N_{m} N_{p}+6\right)^{2}$. Once a time discretization is chosen, Eq. (47) can be cast in the following form

$$
\delta \mathbf{X}^{t+1}=\mathbb{P} \delta \mathbf{X}^{t},
$$

from which it is straightforward to see that a numerical instability arises whenever

$$
\max \left|\lambda_{i}\right|=\left|\lambda_{\max }\right|>1,
$$

where $\lambda_{i}$ are the eigenvalues of $\mathbb{P}$.

In general it does not seem possible to calculate the eigenvalues of $\mathbb{P}$ analytically. Therefore we have performed the analysis numerically. We compare three different time discretizations:

- the explicit first-order accurate Forward Euler (FE) scheme, for which

$$
\mathbb{P}^{F E}=\mathbb{I}+\Delta t \mathbb{A}
$$

- the explicit fourth-order accurate Runge-Kutta (RK4) scheme, for which

$$
\mathbb{P}^{R K 4}=\mathbb{I}+\Delta t \mathbb{A}+\frac{\Delta t^{2}}{2} \mathbb{A}^{2}+\frac{\Delta t^{3}}{6} \mathbb{A}^{3}+\frac{\Delta t^{4}}{24} \mathbb{A}^{4} ;
$$

- the implicit second-order accurate Crank-Nicolson (CN) scheme focus of this paper, for which

$$
\mathbb{P}^{C N}=\left[\mathbb{I}+\frac{\Delta t}{2} \mathbb{A}\right]^{-1}\left[\mathbb{I}-\frac{\Delta t}{2} \mathbb{A}\right] .
$$

The following parameters are considered: $k_{x}=1, k_{y}=k_{z}=0, N_{n}=$ $N_{m}=N_{p}=10, \alpha^{e}=\sqrt{2}, \mathbf{u}^{e}=0, m_{i} / m_{e}=1836, \alpha^{i}=\sqrt{2 m_{e} / m_{i}}, \mathbf{u}^{i}=0$ and $\omega_{p e} / \omega_{c e}=1$. The results are shown in Fig. 1, where $\left|\lambda_{\max }\right|$ is plotted for different values of $\Delta t$ in the range $\Delta t \in\left[10^{-3}, 2\right]$. The forward Euler method is unstable across the whole range of $\Delta t$ and $\left|\lambda_{\max }\right|$ becomes notably larger than one around $\Delta t \sim 0.1$. The Runge-Kutta scheme is stable until $\Delta t \sim 0.5$, while the Crank-Nicholson scheme is stable for any $\Delta t$ considered.

Although the stability analysis discussed in this section is performed numerically and is therefore dependent on the system parameters considered, it confirms the quite obvious fact the implicit discretization of Eqs. (15) and (17) features a wider numerical stability domain than an explicit scheme. In the simulations performed in Sec. 6.3 , this feature will allow us to step over the fastest frequency in the system while retaining a good level of accuracy. 


\section{$5 \quad$ Numerical implementation}

We have developed a serial Matlab code that solves Eqs. (22). For simplicity, we have done it in two spatial dimensions, but retained three components of the velocity. In our code, each unknown is stored as a column vector with dimension $\left(N_{x}+1\right)\left(N_{y}+1\right)$, as shown in expression (21). The total number of unknowns is therefore $N_{t o t}=\left[2 N_{n} N_{m} N_{p}+6\right]\left(N_{x}+1\right)\left(N_{y}+1\right)$, assuming that there are only two plasma species. At each time step the non-linear residual equations (22) are solved with an iterative, inexact Jacobian-Free NewtonKrylov (JFNK) method [34], with initial guess given by the solution at the previous time step. We proceed to discuss the details of the implementation.

\subsection{Jacobian-Free Newton-Krylov solver}

The non-linear system of Eqs. $\mathbf{G}(\mathbf{X})=0$ derived in Sec. 2 is solved with an inexact JFNK method [35]. This is done iteratively by solving successive linear systems of the form

$$
\left.\frac{\partial \mathbf{G}}{\partial \mathbf{X}}\right|_{n} \delta \mathbf{X}^{n}=\mathbb{J}_{n} \delta \mathbf{X}^{n}=-\mathbf{G}\left(\mathbf{X}^{n}\right)
$$

where $n$ labels the (inner) iteration and $\mathbf{X}^{n+1}=\mathbf{X}^{n}+\delta \mathbf{X}^{n}$. Non-linear convergence is reached when

$$
\left\|\mathbf{G}\left(\mathbf{X}^{n}\right)\right\|_{2} \leq \tau_{a}+\tau_{r}\left\|\mathbf{G}\left(\mathbf{X}^{0}\right)\right\|_{2}=\tau_{t}
$$

with $\|\cdot\|$ the $L_{2}$-norm and $\tau_{r}$ and $\tau_{a}$ are relative and absolute tolerances that are provided by the user. For the results presented in this paper, we set $\tau_{a}=\tau_{r}$ and change it parametrically to investigate its impact on the results.

We use a Jacobian-free implementation to solve the linear systems (53), that is, the Jacobian matrix $\mathbb{J}_{n}$ is never formed explicitly and its action on $\delta \mathbf{X}$ is approximated with a directional derivative with a small step increment. This approach reduces the storage requirements of the algorithm. The convergence criteria for the linear iterations is adjusted at each Newton iteration by

$$
\left\|\mathbb{J}_{n} \delta \mathbf{X}^{n}+\mathbf{G}\left(\mathbf{X}^{n}\right)\right\|_{2} \leq \eta_{n}\left\|\mathbf{G}\left(\mathbf{X}^{n}\right)\right\|_{2}
$$

where $\eta_{n}$ is a forcing parameter. In order to avoid volatile decreases in $\eta_{n}$ and oversolving, the sequence of $\eta_{k}$ is prescribed according to [35] 


$$
\begin{aligned}
& \eta_{n}^{A}=\gamma\left(\frac{\left\|\mathbf{G}\left(\mathbf{X}^{n}\right)\right\|_{2}}{\left\|\mathbf{G}\left(\mathbf{X}^{n-1}\right)\right\|_{2}}\right)^{\alpha}, \\
& \eta_{n}^{C}= \begin{cases}\eta_{\max }, & n=0, \\
\min \left(\eta_{\max }, \eta_{n}^{A}\right), & n>0, \gamma \eta_{n-1}^{2} \leq 0.1, \\
\min \left(\eta_{\max }, \max \left(\eta_{n}^{A}, \gamma \eta_{n-1}^{2}\right)\right), & n>0, \gamma \eta_{n-1}^{2} \geq 0.1,\end{cases} \\
& \eta_{n}=\min \left(\eta_{\max }, \max \left(\eta_{n}^{C}, \frac{1}{2} \frac{\tau_{t}}{\left\|\mathbf{G}\left(\mathbf{X}^{k}\right)\right\|_{2}}\right)\right) .
\end{aligned}
$$

Here we fix $\alpha=2, \gamma=0.9$ and $\eta_{\max }=0.9$. Finally, the linear systems (53) are solved with the Generalized Minimum RESidual (GMRES) algorithm [36].

For the Matlab implementation of the JFNK solver, we have used the routines (nsolgm.m, fdgmres.m, givapp.m and dirder.m) that are associated with Ref. [35] and are available on the related website.

\subsection{Preconditioning}

In order to improve the convergence of the inner linear system (53), we consider an alternative system rescaled by right preconditioning,

$$
\mathbb{J}_{n} \mathbb{P}^{-1} \delta \mathbf{Y}^{n}=-\mathbf{G}\left(\mathbf{X}^{n}\right)
$$

where $\mathbb{P}^{-1}$ is the preconditioner matrix and $\delta \mathbf{X}^{n}=\mathbb{P}^{-1} \delta \mathbf{Y}^{n}$. Thus, the application of right preconditioning only requires two matrix-vector multiplications and has the potential to drastically reduce the number of iterations if $\mathbb{P}^{-1} \approx \mathbb{J}_{n}^{-1}$.

In this paper we investigate two preconditioning strategies. In the first one, the preconditioner matrix is the inverse of the matrix arising from the linear terms in Eqs. (22), that is

$$
\mathbb{P}_{1}^{-1}=\left[\begin{array}{cc}
\mathbb{I}-\frac{\Delta t}{2} \mathbb{L}_{1} & \mathbb{O} \\
-\frac{\Delta t}{2} \mathbb{L}_{2} & \mathbb{I}-\frac{\Delta t}{2} \mathbb{L}_{3}
\end{array}\right]^{-1}=\mathbb{A}^{-1}
$$

where $\mathbb{I}$ is the identity matrix and $\mathbb{O}$ is a matrix with all zeroes. We construct matrix $\mathbb{A}$ and obtain its factorization by an LU decomposition, $\mathbb{A}=\mathbb{L} \mathbb{U}$. Then we set $\mathbb{P}^{-1}=\mathbb{U}^{-1} \mathbb{L}^{-1}$. [We have also tried an incomplete LU (ILU) decomposition such that $\mathbb{A} \approx \mathbb{L} \mathbb{U}$ (using Matlab ILU routine with tolerance set to $10^{-6}$ ), without much difference for the results presented in Sec. 6].

In the second one, we improve upon $\mathbb{P}_{1}^{-1}$ by accounting for the convolutions 
in Eq. (22) via linearization relative to the solution at the previous time step

$$
\mathbb{P}_{2}^{-1}=\left[\begin{array}{rc}
\mathbb{I}-\frac{\Delta t}{2} \mathbb{L}_{1}-\frac{\Delta t}{2} \mathcal{N}\left(\cdot, \mathbf{F}^{n}\right) & -\frac{\Delta t}{2} \mathcal{N}\left(\mathbf{C}^{n}, \cdot\right) \\
-\frac{\Delta t}{2} \mathbb{L}_{2} & \mathbb{I}-\frac{\Delta t}{2} \mathbb{L}_{3}
\end{array}\right]^{-1}=\mathbb{B}^{-1}
$$

The choice of linearization relative to previous time step and not to the previous iterate of the Newton sequence is motivated by the necessity of keeping the cost of constructing the preconditioner low. At each time step, we construct $\mathbb{B}$ (keeping only entries such that $\left|\mathbf{X}^{n}\right|>10^{-1}$ ) and use an incomplete LU

factorization (with tolerance to be specified) to calculate $\mathbb{B}^{-1}$. We note that a tigher ILU tolerance results in a more effective preconditioner which, however, could be much more costly to construct than that for a looser tolerance. Similarly, one could also envision variations of $\mathbb{P}_{2}^{-1}$ where the convolution terms are evaluated only for a subset of moments (for instance the first three in each direction, since these are related to well known macroscopic quantities such as density, momentum and energy [31]). This kind of preconditioning would be similar to the moment acceleration discussed in Refs. [28,29]. In general the best preconditioning strategy requires a trade-off between the cost of constructing the preconditioner and its efficiency in reducing the number of linear iterations, and depends on the specific application. In Sect. 6 we will compare the performance of $\mathbb{P}_{1}^{-1}$ and $\mathbb{P}_{2}^{-1}$ to obtain some general guidance, and leave the search for the optimal preconditioner to future work.

We note that preconditioner $\mathbb{P}_{1}^{-1}$ is rather simple, but has the advantage that it can be computed just once at the beginning of the simulation. For the applications discussed in this paper, it provides a reasonable choice for cases where one does not need to step over the fastest scales and in our examples has reduced the number of linear iteration by $\sim 50 \%$ relative to the unpreconditioned case. Preconditioner $\mathbb{P}_{2}^{-1}$ requires much more time for its construction (which is performed at every time step) but has proven to be very effective in cases where one can afford larger time steps (see Sec. 6), reducing by more than an order of magnitude the number of linear iterations relative to the unpreconditioned case and achieving a factor of six in code speed-up.

\subsection{Exploiting symmetries of the Fourier series}

Since we are interested in physics applications where the relevant quantities are real, it is possible to exploit the properties of the Fourier series to reduce the number of unknowns in the system. In two spatial dimensions, one has

$$
H\left(k_{x}, k_{y}\right)=H^{*}\left(-k_{x},-k_{y}\right)
$$


where ${ }^{*}$ denotes complex conjugation. Therefore, one only needs to allocate about half of the $\left(k_{x}, k_{y}\right)$ spectrum, for instance $k_{x}=\left[-\frac{N_{x}}{2}, \ldots, \frac{N_{x}}{2}\right]$ and $k_{y}=$ $\left[0, \ldots, \frac{N_{y}}{2}\right]$, and can reconstruct the other half (which is necessary to evaluate convolutions) by means of property (60).

We have implemented both options, where we allocate the full spectrum or half of it, in Matlab. It is interesting to note that, in our Matlab application, allocating the full spectrum provides a faster code. This is because in the second option some sizeable fraction of time is spent manipulating matrices to reconstruct the full spectrum. Of course, allocating only part of the spectrum could be important in production run applications that might require a large number of modes or moments.

Last, we note that convolutions can be handled very efficiently via the Fast Fourier Transform (FFT), with an overal scaling $\propto N \log N$ (where $N$ is the number of terms in the convolution). We have tried both a regular convolution (scaling as $N^{2}$ ) and the FFT-based convolution. The former is faster for small problems, but the latter will be necessary for large systems.

\subsection{Collisional operator}

A common feature of collisionless plasmas is the development of finer and finer scales in phase space, usually referred to as filamentation. From the perspective of the Hermite basis, this implies that higher order modes are progressively excited until the simulation runs out of resolution because of the truncation in expansion (8), and this can lead to numerical instabilities [21]. It can also lead to the well known recurrence effect, where the initial distribution is artificially recreated after a certain amount of time because of the finite resolution in velocity space, and the simulation results are then compromised without any instability $[3,37,38]$. For this reason, it is common practice to introduce a collision operator to damp higher order modes. Here, we use the collision operator introduced in Ref. [21] (extended to three dimensions) and add the following term to the right hand side of Eq. (15)

$$
\begin{gathered}
\mathcal{C}\left[C_{n, m, p}^{k_{x}, k_{y}, k_{z}, s}\right]=-\nu\left[\frac{n(n-1)(n-2)}{N_{n}\left(N_{n}-1\right)\left(N_{n}-2\right)}+\frac{m(m-1)(m-2)}{N_{m}\left(N_{m}-1\right)\left(N_{m}-2\right)}+\right. \\
\left.\frac{p(p-1)(p-2)}{N_{p}\left(N_{p}-1\right)\left(N_{p}-2\right)}\right] C_{n, m, p}^{k_{x}, k_{y}, k_{z}, s}
\end{gathered}
$$


where $\nu$ is a collisional rate. Note that Eq. (61) does not act on the first three Hermite modes (in each direction) and therefore preserves the conservation laws derived in Sec. 3.

Although our numerical implementation is serial, it is worth discussing some strategies for its parallelization. The FFT-based convolutions are the most computationally intensive part of the residual function evaluation. Also, it is well known that FFT does not scale well on distributed memory systems. On such systems, a simple strategy would be to perform the domain decomposition only in velocity space to avoid splitting the FFT among processes. By storing a copy of the electromagnetic field on each process, the FFT-based convolution could be performed without any communication. This strategy limits the spatial domain size that could be handled, but the memory redundancy overhead would be tolerable for moderately large problem sizes. For instance, storing six complex fields in double precision on a $512^{2}$ grid requires only $\sim 23 \mathrm{Mb}$ of memory. Also, the solver can be easily parallelized since the only change relative to its serial implementation is the communication of $\mathrm{C}^{s}$ boundary values between processes. Communication time could be hidden by using non-blocking communications. An important issue would be the scalability of the preconditioner, and one could change the ILU preconditioner discussed in this paper to leverage those supported by parallel libraries like PETSc, Trillinos or others.

\section{Results}

In this Section, we perform some standard tests to benchmark our FourierHermite code following Ref. [19]. The simulations start from an equilibrium configuration where the plasma is described by a Maxwellian distribution function along each direction

$f_{s}=\frac{1}{(2 \pi)^{3 / 2}} \frac{1}{v_{t h, x}^{s} v_{t h, y}^{s} v_{t h, z}^{s}} \exp \left[-\frac{\left(v_{x}-u_{x}^{s}\right)^{2}}{2 v_{t h, x}^{s}}\right] \exp \left[-\frac{\left(v_{y}-u_{y}^{s}\right)^{2}}{2 v_{t h, y}^{s}}\right] \exp \left[-\frac{\left(v_{z}-u_{z}^{s}\right)^{2}}{2 v_{t h, z}^{s}}\right]$

where $v_{t h, \beta}^{s}=\sqrt{T_{\beta}^{s} / m_{s}}$ and $T_{\beta}^{s}$ is the temperature of species $s$ along direction $\beta$. The system is then perturbed (as described separately for each test case) to induce dynamics. Note that Eq. (62) implies that the plasma equilibrium can be represented by using only the first moment of the Hermite expansion

$$
C_{0,0,0}^{0,0,0, s}=\frac{1}{\alpha^{s}}
$$


with $\alpha_{\beta}^{s}=\sqrt{2} v_{t h, \beta}^{s}$. In what follows we only consider cases where $u_{\beta}^{s}=0$.

\subsection{Convergence study on a Landau damping test}

We begin by performing a convergence study on the same Landau damping problem for which the numerical stability of our scheme was proven in Sec. 4. Landau damping is a standard test of kinetic theory for collisionless plasmas: a wave initially excited in the plasma interacts efficiently with particles that are resonant with it. Since in a Maxwellian plasma there are more particles gaining energy from than losing energy to the wave, the wave damps. We consider the following parameters: $N_{x}=16, N_{y}=0, N_{n}=N_{m}=N_{p}=10, \alpha^{e}=\sqrt{2}$, $\mathbf{u}^{e}=0, m_{i} / m_{e}=1836, \alpha^{i}=\sqrt{2 m_{e} / m_{i}}, \mathbf{u}^{i}=0$ and $\omega_{p e} / \omega_{c e}=1$. We also fix $\nu=10$ while $L_{x}=L_{y}=L_{z}=2 \pi$. At time $\omega_{p e} t=0$, we excite a wave by perturbing the electron density: $C_{0,0,0}^{1,0,0, e}=\varepsilon \cos (x) / \alpha^{e}$, with $\varepsilon=10^{-2}$. Consistently, an initial electric field is initialized as $E_{x}^{1,0,0}=-\varepsilon \sin (x)$. The total length of the simulation is $\omega_{p e} T=10$.

We perform a convergence study by varying the time step of the simulation in the interval $\omega_{p e} \Delta t \in[0.03125-1]$. We compare against a reference solution obtained by solving the linearized system given by Eq. (47) with the exponential matrix technique calculated for a small time step $\Delta t^{r e f}=0.001$ (superscript 'ref' labels the reference solution). We define the following measure of error

$$
\text { Error }=\frac{\int_{0}^{\omega_{p e} T}\left|E_{x}^{1,0,0}(t)-E_{x}^{1,0,0, \text { ref }}(t)\right| d t}{\int_{0}^{\omega_{p e} T}\left|E_{x}^{1,0,0, r e f}(t)\right| d t} .
$$

Figure 2 (left) shows the time evolution of the absolute value of $E_{x}^{1,0,0}$ for a simulation with $\omega_{p e} \Delta t=0.03125$ (solid line), compared successfully against the reference solution (o). One can see that the theoretical Landau damping rate $\gamma_{\text {theory }} / \omega_{\text {pe }}=-0.85$ is recovered (dashed line): the slope between the second and fourth peak of $\left|E_{x}^{1,0,0}\right|$ is approximately $\gamma_{s i m} / \omega_{p e}=-0.83$. Figure 2 (right) shows the error as a function of $\Delta t$. The theoretical scaling $\propto \Delta t^{2}$ expected from the second order accurate Crank-Nicolson scheme is recovered: using a lest squares fit of the data in the range $\omega_{p e} \Delta t=[0.03125-0.125]$ yields Error $\propto \Delta t^{2.02}$. The convergence analysis in terms of the Hermite expansion on a Landau damping test can be found in Ref. [22]. 


\subsection{High frequency waves in a magnetized plasma}

We now study a system characterized by a constant background magnetic field $\mathbf{B}_{0}=\left(B_{0 x}, B_{0 y}, B_{0 z}\right)=(1,0,0)$ and study the propagation of high frequency waves (i.e. waves for which ion dynamics is not important) in the plasma. We use the same parameters that were considered in Ref. [19]. Specifically, we have $\omega_{p e} / \omega_{c e}=4, m_{i} / m_{e}=1836, T_{x}^{s}=T_{y}^{s}=T_{z}^{s}, T_{\beta}^{i} / T_{\beta}^{e}=100$ and $v_{t h, \beta}^{e}=0.02$. For these parameters, the plasma characteristic modes are well described by cold plasma theory and the dispersion relation is given by [39]

$$
\frac{k^{2} c^{2}}{\omega^{2}}=1-\frac{1}{\omega^{2}} \frac{2 \omega_{p e}^{2}\left(\omega^{2}-\omega_{p e}^{2}\right)}{2\left(\omega^{2}-\omega_{p e}^{2}\right)-\omega_{c e}^{2} \sin ^{2} \theta \pm \omega_{c e} \Delta}
$$

where $\Delta=\sqrt{\omega_{c e}^{2} \sin ^{4} \theta+4\left(\omega^{2}-\omega_{p e}^{2}\right)^{2} \cos ^{2} \theta / \omega^{2}}$. In Eq. (65), $\omega$ is the frequency of the mode and $\theta$ is the angle between the background magnetic field and the wave vector $k$.

For waves propagating parallel to the background magnetic field, our simulation parameters are $N_{x}=32, N_{y}=0, N_{n}=N_{m}=N_{p}=5, \nu=0$, $\Delta t=0.2$ and the final simulation time is $T=1280$. The system size is $L_{x}=L_{y}=L_{z}=20 \pi$. At time $t=0$, the system is perturbed by initializing a small amplitude electron current along $z$ for each $k_{x} \neq 0$ mode (i.e. we perturb $\left.C_{0,0,1}^{k_{x}, 0,0, e}\right), J_{z}^{k_{x}, 0,0, e}=\varepsilon \cos \left(k_{x} x\right)$, with $\varepsilon=10^{-4}$. Since the system is not in equilibrium at time $t=0$, it evolves by exciting the characteristic modes of the plasma. Figure 3 shows the wave spectrum for $\log _{10}\left|\tilde{E}_{y}\left(k_{x}, \omega\right)\right|$, obtained by applying the Fast Fourier Transform (FFT) to the real part of $E_{y}^{k_{x}, 0,0}(t)$, i.e. $\tilde{E}_{y}\left(k_{x}, \omega\right)=\operatorname{FFT}\left[\operatorname{Re} E_{y}^{k_{x}, 0,0}(t)\right]$. One can clearly see that the wave energy is concentrated in the characteristic modes of the plasma. These are the right $(\mathrm{R})$ and left $(\mathrm{L})$ hand circularly polarized waves that can be obtained from Eq. (65) with $\theta=0$ (the + sign corresponds to the $\mathrm{L}$ wave, while the - sign gives the two $\mathrm{R}$ waves, including the whistler branch for $\omega<\omega_{c e}=0.25$ ) and that are marked with black solid lines in Fig. 3. Thus, the dispersion relation (65) is verified numerically. For these runs, we have used preconditioner $\mathbb{P}_{1}^{-1}$. The average number of linear (non-linear) iterations is 7 (5.2). The absolute changes in momentum, $\Delta P_{\beta}=P_{\beta}-P_{\beta}(0)$, are $\left|\Delta P_{x}\right|<2 \cdot 10^{-17}$, $\left|\Delta P_{y}\right|<4 \cdot 10^{-14}$ and $\left|\Delta P_{z}\right|<7 \cdot 10^{-14}$. The relative variation of total energy $\Delta W / W(0)=(W-W(0)) / W(0)$ is $|\Delta W| / W(0)<10^{-14}$.

Next, we study waves that are propagating perpendicularly to $\mathbf{B}_{0}$. The parameters are as before except that now $N_{x}=0$ and $N_{y}=32$. The system is perturbed at time $t=0$ by introducing an electron current along $x$ and $z$ for each $k_{y} \neq 0$ mode, $J_{x}^{0, k_{y}, 0, e}=J_{z}^{0, k_{y}, 0, e}=\varepsilon \cos \left(k_{y} y\right)$, such that plasma modes with electric field parallel and perpendicular to the background magnetic field 
can be excited. Figure 4 shows the wave spectrum for $\log _{10}\left|\tilde{E}_{x}\left(k_{y}, \omega\right)\right|$ (left) and $\log _{10}\left|\tilde{E}_{y}\left(k_{y}, \omega\right)\right|$ (right), together with the dispersion relation (65) with $\theta=\pi / 2$ plotted with black solid lines. Once again, the dispersion relation (65) is verified numerically and one can see that the wave energy is concentrated in the ordinary $(\mathrm{O})$ mode in Fig. 4 (left) and in the slow and fast extraordinary (X) modes in Fig. 4 (right). For these runs, we have again used preconditioner $\mathbb{P}_{1}^{-1}$. The average number of linear (non-linear) iterations is 7 (5). Again, the conservation laws are satisfied numerically: $\left|\Delta P_{x}\right|<5 \cdot 10^{-13}$, $\left|\Delta P_{y}\right|<2 \cdot 10^{-11},\left|\Delta P_{z}\right|<7 \cdot 10^{-11}$, and $|\Delta W| / W(0)<5 \cdot 10^{-14}$.

\subsection{Whistler instability}

Next, we study the case of a whistler instability driven by electron temperature anisotropy. The whistler instability occurs commonly in space plasmas. For instance, it is a mechanism by which low energy electrons injected in the inner magnetosphere excite whistler (chorus) waves [40,41]. The latter are extremely important for the dynamics of the ring current and radiation belts via wave-particle interaction processes.

The chosen plasma parameters are typical of the inner magnetosphere: $\omega_{p e} / \omega_{c e}=$ $4, m_{i} / m_{e}=1836, T_{y}^{e}=T_{z}^{e}=T_{\beta}^{i}, T_{y}^{e}=5 T_{x}^{e}, v_{t h, x}^{e}=0.0559$. The system is characterized by a constant background magnetic field $\mathbf{B}_{0}=\left(B_{0 x}, B_{0 y}, B_{0 z}\right)=$ $(1,0,0)$. For these conditions, the maximum growth rate of the whistler instability occurs for $k_{y}=0$. Therefore our simulations parameters are $N_{x}=32$, $N_{y}=0, N_{n}=N_{m}=N_{p}=10, L_{x}=L_{y}=L_{z}=2 \pi$. Note that we have set $\nu=1$ to avoid filamentation problems. The system is perturbed at $t=0$ by introducing an electron current along $z, J_{z}^{e}=\varepsilon \cos (x)$, with $\varepsilon=10^{-3}$. The total simulation time is $T=200$.

Figure 5 shows the time evolution of $\left|B_{y}^{1,0,0}\right|,\left|B_{z}^{1,0,0}\right|$ and $\left|B^{1,0,0}\right|=\sqrt{\left|\left(B_{x}^{1,0,0}\right)^{2}+\left(B_{y}^{1,0,0}\right)^{2}+\left(B_{z}^{1,0,0}\right)^{2}\right|}$ obtained for $\Delta t=0.2$. After an initial oscillatory transient, the system becomes unstable to the whistler instability and all curves begin to grow. Saturation occurs at $t \sim 150$. Figure 5 also shows the curve $\propto \exp \left(\gamma_{\text {theory }} t\right)$ with a dashed line, where the theoretical growth rate $\gamma_{\text {theory }}$ has been calculated with the WHAMP code [42]. There is good agreement between theory and simulations: $\gamma_{\text {theory }} / \omega_{p e}=0.034$ compared to $\gamma_{s i m} / \omega_{p e}=0.033$, indicating that the chosen level of resolution is adequate for the linear phase. Figure 6 shows the time evolution of $\left|B^{1,0,0}\right|$ changing $\Delta t$, obtained for $\Delta t=0.2,0.5,1,2$. There is a remarkable agreement between all the curves in terms of growth rate and saturation, indicating that our fully implicit approach can step over the electron plasma frequency without incurring in numerical instabilities.

Figure 7 (a)-(d) shows how well the conservation laws derived in Sec. 
3 are satisfied numerically as a function of the non-linear tolerance of the solver $\tau_{a}=\tau_{r}$, for the simulations with $\Delta t=2$ and using preconditioner $\mathbb{P}_{2}^{-1}$. Figure 7 (e) also shows the numerical verification of Gauss' law (4) (recall that the Fourier discretization employed in this paper ensures that Gauss' law will be satisfied at all times if it is satisfied at time $t=0$ ), while Figure 7 (f) shows the maximum error on the constraint $\nabla \cdot \mathbf{B}=0$. In general one can notice that the conservation laws, Gauss' law and the $\nabla \cdot \mathbf{B}=0$ constraint are indeed satisfied in the numerical experiments, to a degree that is controlled by the tolerance of the non-linear solver. On this one-dimensional example, we have also verified that the total canonical angular momentum (whose exact conservation in the ignorable directions has been recently achieved in the context of PIC [43]) in the $y$ direction (gauge $A_{x}=0$, where $\mathbf{A}$ is the vector potential) is conserved numerically with a degree of accuracy similar to that of $\left|\Delta P_{y}\right|$. Finally, entropy is not conserved as expected due to the collisional operator necessary to damp filamentation. On this example entropy grows in time, with a maximum relative error (for the electron species) of about $4 \%$.

We have used this example to test the performance of the preconditioner strategies discussed in Sec. 5.2. The results are presented in Table 1, where runs with and without preconditioning are compared in terms of number of non-linear (Newton) and linear (GMRES) iterations averaged over the entire simulation, and of code speed-up. The latter is defined in Table 1 as the CPU time necessary to complete the simulation normalized to that for the unpreconditioned case with $\Delta t=0.2$. Relative to the unpreconditioned case, one can see that preconditioner $\mathbb{P}_{1}^{-1}$ reduces the number of linear iterations by $\sim 50 \%$ for $\Delta t=0.2$ and by $\sim 75 \%$ for $\Delta t=2$, resulting in a net performance gain. Even though its performance becomes worse as $\Delta t$ increases and it takes about 6 times more linear iterations for $\Delta t=2$ than for $\Delta t=0.2$, preconditioned $\mathbb{P}_{2}^{-1}$ is surprisingly effective also for the larger $\Delta t$. Preconditioner $\mathbb{P}_{2}^{-1}$ (with Matlab ILU droptol $=10^{-8}$ ), on the other hand, reduces the number of linear iterations by more than an order of magnitude relative to the unpreconditioned case and achieves a factor of six gain in code speedup. Its construction and inversion, however, is quite costly and a net gain in performance is only achieved for the larger $\Delta t=2$.

Finally, we study a two-dimensional example of the whistler instability. The physical parameters are the same as in the previous example, but we now have $N_{x}=N_{y}=16$ and $N_{n}=N_{m}=N_{p}=$ 12. We initialize a perturbation in the $z$ electron current equal to $J_{z}^{e}=2 \varepsilon \cos (x) \cos (y)$, with $\varepsilon=10^{-4}$. We have chosen $\tau_{a}=\tau_{r}=10^{-6}$, $\Delta t=0.25$ and the total simulation time is $T=460$. The system is linearly unstable with growth rate $\gamma_{\text {theory }} / \omega_{\text {pe }}=0.018$ obtained by the 


\begin{tabular}{|c|c|c|c|c|}
\hline$\Delta t$ & Preconditioner & Average Newton iterations & Average GMRES iterations & $\frac{\text { CPU time }}{\text { CPU time }(\Delta t=0.2, \text { unprec })}$ \\
\hline 0.2 & $\mathbb{I}$ & 5.3 & 14.5 & 1 \\
0.2 & $\mathbb{P}_{1}^{-1}$ & 5.3 & 7.3 & 0.65 \\
2 & $\mathbb{I}$ & 7.6 & 209.6 & 1.07 \\
2 & $\mathbb{P}_{1}^{-1}$ & 6.2 & 48.8 & 0.27 \\
2 & $\mathbb{P}_{2}^{-1}$ & 5.4 & 9.8 & 0.18 \\
\hline
\end{tabular}

Table 1

Whistler instability: performance of the preconditioner.

WHAMP code. Figure 8 (left) shows the time evolution of $\left|B^{1,1,0}\right|$ (solid line), together with the theoretical growth rate (dashed line). Numerically, we compute $\gamma_{s i m} / \omega_{p e}=0.17$, in good agreement with the theoretical one. Figure 8 (right) shows the time evolution of the relative change of different contributions to the total energy, defined as $\Delta W_{\alpha} / W(0)=\left(W_{\alpha}-W(t=0)\right) / W(t=0)$. For the chosen parameters, the instability involves only a modest (less than $0.1 \%$ ) amount of energy transfer, mostly from the kinetic energy of the electrons to the magnetic field energy. The conservation laws derived in Sec. 3 are indeed satisfied numerically, as shown in Fig. 9. The maximum error on Gauss' law (4) or on the $\nabla \cdot \mathrm{B}=0$ constraint is approximately $10^{-17}$ (not shown).

\section{Conclusions}

In this work, we have presented a spectral method for the numerical solution of the Vlasov-Maxwell equations in multiple dimensions. We have assumed periodic boundary conditions for the spatial coordinates and expanded the plasma distribution function in Fourier (for the spatial part) and Hermite (for the velocity) basis functions. The resulting set of ordinary differential equations for the coefficients (moments) of the expansion is truncated by assuming that all expansion coefficients are zero beyond a fixed number.

We have introduced a Crank-Nicolson, second order accurate, fully implicit time discretization and solved the moment system by means of a preconditioned Jacobian-Free Newton-Krylov technique with GMRES as linear solver. It is shown analytically that the Fourier-Hermite method features exact conservation laws for total mass, momentum and energy in discrete form. Keys to this result are the periodic boundary conditions and the Crank-Nicolson discretization. The validity of the conservation laws is confirmed with numerical experiments involving plasma waves and the whistler instability. Moreover, the whistler instability test shows that, due to the fully implicit time discretization, we can step over the fastest frequency in the system without any sign of numerical instability. Some preconditioning strategies have also been 
presented, showing an order of magnitude decrease in the number of linear iterations and a factor of six gain in code speed-up.

Despite its proof-of-principle nature, this work proposes for the first time a fully implicit spectral method for the Vlasov-Maxwell equations with exact discrete conservation laws. Future work will explore the use of optimization techniques to minimize the number of basis functions necessary for a given accuracy in phase space and how to take advantage of high performance computing architectures. Both aspects are necessary before the method proposed here could really tackle serious applications.

\section{Acknowledgements}

The author gratefully acknowledges discussions with Enrico Camporeale, Luis Chacón, Stefano Markidis, David Moulton, Xian-Zhu Tang and Juris Vencels. This work was funded by the Laboratory Directed Research and Development program (LDRD), under the auspices of the National Nuclear Security Administration of the U.S. Department of Energy by Los Alamos National

Laboratory, operated by Los Alamos National Security LLC under contract DE-AC52-06NA25396.

\section{References}

[1] R. J. Goldston and P. Rutherford, Introduction to plasma physics. Plasma Physics Series, Institute of Physics Pub., 1995.

[2] C. K. Birdsall and A. B. Langdon, Plasma Physics Via Computer Simulation. Taylor \& Francis, 2004.

[3] C. Cheng and G. Knorr, "The integration of the Vlasov equation in configuration space," Journal of Computational Physics, vol. 22, no. 3, pp. 330 $-351,1976$.

[4] E. Sonnendrücker, J. Roche, P. Bertrand, and A. Ghizzo, "The Semi-Lagrangian Method for the Numerical Resolution of the Vlasov Equation," Journal of Computational Physics, vol. 149, no. 2, pp. 201 - 220, 1999.

[5] F. Filbet, E. Sonnendrücker, and P. Bertrand, "Conservative Numerical Schemes for the Vlasov Equation," Journal of Computational Physics, vol. 172, no. 1 , pp. 166 - 187, 2001.

[6] T. Armstrong, R. Harding, G. Knorr, and D. Montgomery, "Solution of Vlasov's equation by transform methods," Methods Comput. Phys., vol. 9, p. 29, 1970. 
[7] M. Shoucri and R. Gagne, "Numerical solution of the Vlasov equation by transform methods," Journal of Computational Physics, vol. 21, no. 2, pp. 238 $-242,1976$.

[8] F. Engelmann, M. Feix, E. Minardi, and J. Oxenius, "Nonlinear Effects from Vlasov's Equation," Physics of Fluids, vol. 6, no. 2, pp. 266-275, 1963.

[9] A. J. Klimas, "A numerical method based on the Fourier-Fourier transform approach for modeling 1-d electron plasma evolution," Journal of Computational Physics, vol. 50, no. 2, pp. 270 - 306, 1983.

[10] J. P. Holloway, "Spectral velocity discretizations for the Vlasov-Maxwell equations," Transport Theory and Statistical Physics, vol. 25, no. 1, pp. 1-32, 1996.

[11] J. W. Schumer and J. P. Holloway, "Vlasov Simulations Using Velocity-Scaled Hermite Representations," Journal of Computational Physics, vol. 144, no. 2, pp. $626-661,1998$.

[12] J. Banks and J. Hittinger, "A New Class of Nonlinear Finite-Volume Methods for Vlasov Simulation," Plasma Science, IEEE Transactions on, vol. 38, pp. 2198-2207, Sept 2010.

[13] N. Sircombe and T. Arber, "VALIS: A split-conservative scheme for the relativistic 2D Vlasov-Maxwell system," Journal of Computational Physics, vol. 228 , no. 13 , pp. $4773-4788,2009$.

[14] S. Maeyama, A. Ishizawa, T.-H. Watanabe, N. Nakajima, S. Tsuji-Iio, and H. Tsutsui, "A hybrid method of semi-Lagrangian and additive semi-implicit Runge-Kutta schemes for gyrokinetic Vlasov simulations," Computer Physics Communications, vol. 183, no. 9, pp. 1986 - 1992, 2012.

[15] K. Imadera, Y. Kishimoto, D. Saito, J. Li, and T. Utsumi, "A numerical method for solving the Vlasov-Poisson equation based on the conservative IDO scheme," Journal of Computational Physics, vol. 228, no. 23, pp. 8919 - 8943, 2009.

[16] N. Crouseilles, T. Respaud, and E. Sonnendrücker, "A forward semi-Lagrangian method for the numerical solution of the Vlasov equation," Computer Physics Communications, vol. 180, no. 10, pp. 1730 - 1745, 2009.

[17] A. Christlieb, W. Guo, M. Morton, and J.-M. Qiu, "A high order time splitting method based on integral deferred correction for semi-Lagrangian Vlasov simulations," Journal of Computational Physics, vol. 267, no. 0, pp. 7 27, 2014.

[18] S. L. Bourdiec, F. de Vuyst, and L. Jacquet, "Numerical solution of the VlasovPoisson system using generalized Hermite functions," Computer Physics Communications, vol. 175, no. 8, pp. 528 - 544, 2006.

[19] B. Eliasson, "Outflow boundary conditions for the Fourier transformed three-dimensional Vlasov-Maxwell system," Journal of Computational Physics, vol. 225 , no. 2 , pp. $1508-1532,2007$. 
[20] B. Eliasson, "Numerical Simulations of the Fourier-Transformed VlasovMaxwell System in Higher Dimensions-Theory and Applications," Transport Theory and Statistical Physics, vol. 39, no. 5-7, pp. 387-465, 2010.

[21] E. Camporeale, G. L. Delzanno, B. K. Bergen, and J. D. Moulton, "On the velocity space discretization for the Vlasov-Poisson system: comparison between hermite spectral and Particle-in-Cell methods. part 1: semi-implicit scheme," under review, 2014.

[22] E. Camporeale, G. L. Delzanno, B. K. Bergen, and J. D. Moulton, "On the velocity space discretization for the Vlasov-Poisson system: comparison between Hermite spectral and Particle-in-Cell methods. part 2: fully-implicit scheme," under review, 2014.

[23] H. Lewis, "Energy-conserving numerical approximations for vlasov plasmas," Journal of Computational Physics, vol. 6, no. 1, pp. 136 - 141, 1970.

[24] E. Evstatiev and B. Shadwick, "Variational formulation of particle algorithms for kinetic plasma simulations," Journal of Computational Physics, vol. 245, no. 0 , pp. $376-398,2013$.

[25] S. Markidis and G. Lapenta, "The energy conserving particle-in-cell method," Journal of Computational Physics, vol. 230, no. 18, pp. 7037 - 7052, 2011.

[26] G. Chen, L. Chacón, and D. Barnes, "An energy- and charge-conserving, implicit, electrostatic particle-in-cell algorithm," Journal of Computational Physics, vol. 230, no. 18, pp. 7018 - 7036, 2011.

[27] Y. Cheng, A. J. Christlieb, and X. Zhong, "Energy-conserving discontinuous Galerkin methods for the VlasovAmpere system," Journal of Computational Physics, vol. 256, no. 0, pp. $630-655,2014$.

[28] W. T. Taitano and L. Chacón, "Charge-and-Energy Conserving Moment-Based Accelerator for a Multi-Species Vlasov-Fokker-Planck-Ampère System, Part I: Collisionless Aspects," under review for Journal of Computational Physics, 2014.

[29] W. T. Taitano, D. A. Knoll, and L. Chacón, "Charge-and-Energy Conserving Moment-Based Accelerator for a Multi-Species Vlasov-FokkerPlanck-Ampère System, Part II: Collisional Aspects," under review for Journal of Computational Physics, 2014.

[30] H. Grad, "On the kinetic theory of rarefied gases," Communications on pure and applied mathematics, vol. 2, no. 4, pp. 331 - 407, 1949.

[31] E. Camporeale, G. L. Delzanno, G. Lapenta, and W. Daughton, "New approach for the study of linear Vlasov stability of inhomogeneous systems," Physics of Plasmas, vol. 13, no. 9, pp. -, 2006.

[32] I. S. Gradshteyn and I. M. Ryzhik, Table of integrals, series, and products. Elsevier/Academic Press, Amsterdam, Seventh ed., 2007. 
[33] J. Crank and P. Nicolson, "A practical method for numerical evaluation of solutions of partial differential equations of the heat-conduction type," Mathematical Proceedings of the Cambridge Philosophical Society, vol. 43, pp. 50-67, 11947.

[34] D. Knoll and D. Keyes, "Jacobian-free Newton-Krylov methods: a survey of approaches and applications," Journal of Computational Physics, vol. 193, no. 2, pp. $357-397,2004$.

[35] C. T. Kelley, Iterative Methods for Linear and Nonlinear Equations. SIAM, Philadelphia, 1995.

[36] Y. Saad and M. Schultz, "GMRES: A generalized minimal residual algorithm for solving nonsymmetric linear systems," SIAM Journal on Scientific and Statistical Computing, vol. 7, no. 3, pp. 856-869, 1986.

[37] G. Joyce, G. Knorr, and H. K. Meier, "Numerical integration methods of the vlasov equation," Journal of Computational Physics, vol. 8, no. 1, pp. 53 -63, 1971.

[38] J. Canosa, J. Gazdag, and J. Fromm, "The recurrence of the initial state in the numerical solution of the vlasov equation," Journal of Computational Physics, vol. 15, no. 1, pp. $34-45,1974$.

[39] T. Stix, Waves in Plasmas. American Inst. of Physics, 1992.

[40] S. P. Gary, Theory of Space Plasma Microinstabilities. Cambridge University Press, 2005.

[41] V. K. Jordanova, R. M. Thorne, W. Li, and Y. Miyoshi, "Excitation of whistler mode chorus from global ring current simulations," Journal of Geophysical Research: Space Physics, vol. 115, no. A5, pp. n/a-n/a, 2010.

[42] K. Rnnmark, "Whamp-waves in homogeneous, anisotropic multicomponent plasmas," Kiruna Geophysical Institute Report, p. 179, 1982.

[43] G. Chen and L. Chacón, "An energy- and charge-conserving, nonlinearly implicit, electromagnetic 1D-3V Vlasov-Darwin particle-in-cell algorithm," Computer Physics Communications, vol. 185, no. 10, pp. 2391 - 2402, 2014. 


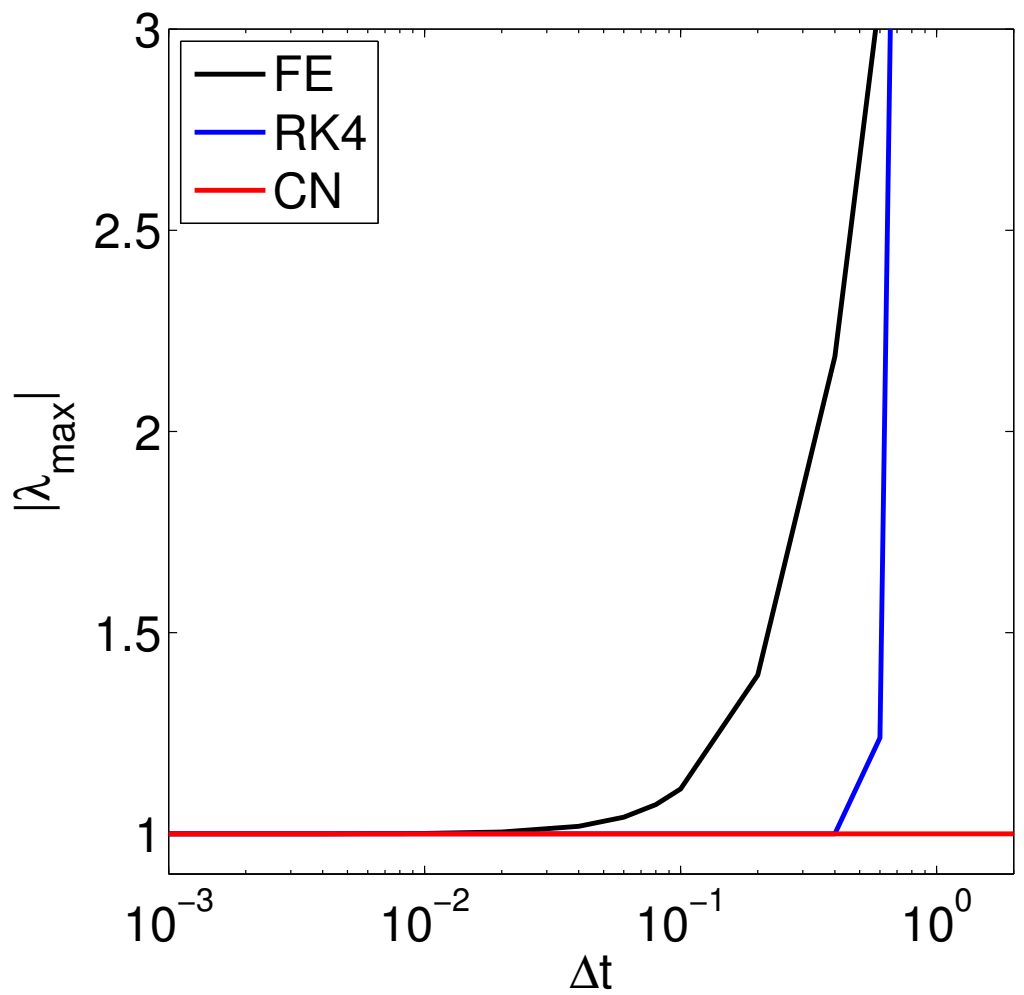

Fig. 1. Absolute value of the maximum eigenvalue of matrix $\mathbb{P}$ for three different time discretizations [forward Euler (black), $4^{\text {th }}$ order Runge-Kutta (blue) and Crank-Nicolson (red)] versus time step $\Delta t$. The system parameters are: $k_{x}=1, k_{y}=k_{z}=0$, $N_{n}=N_{m}=N_{p}=10, \alpha^{e}=\sqrt{2}, \mathbf{u}^{e}=0, m_{i} / m_{e}=1836, \alpha^{i}=\sqrt{2 m_{e} / m_{i}}, \mathbf{u}^{i}=0$ and $\omega_{p e} / \omega_{c e}=1$. 

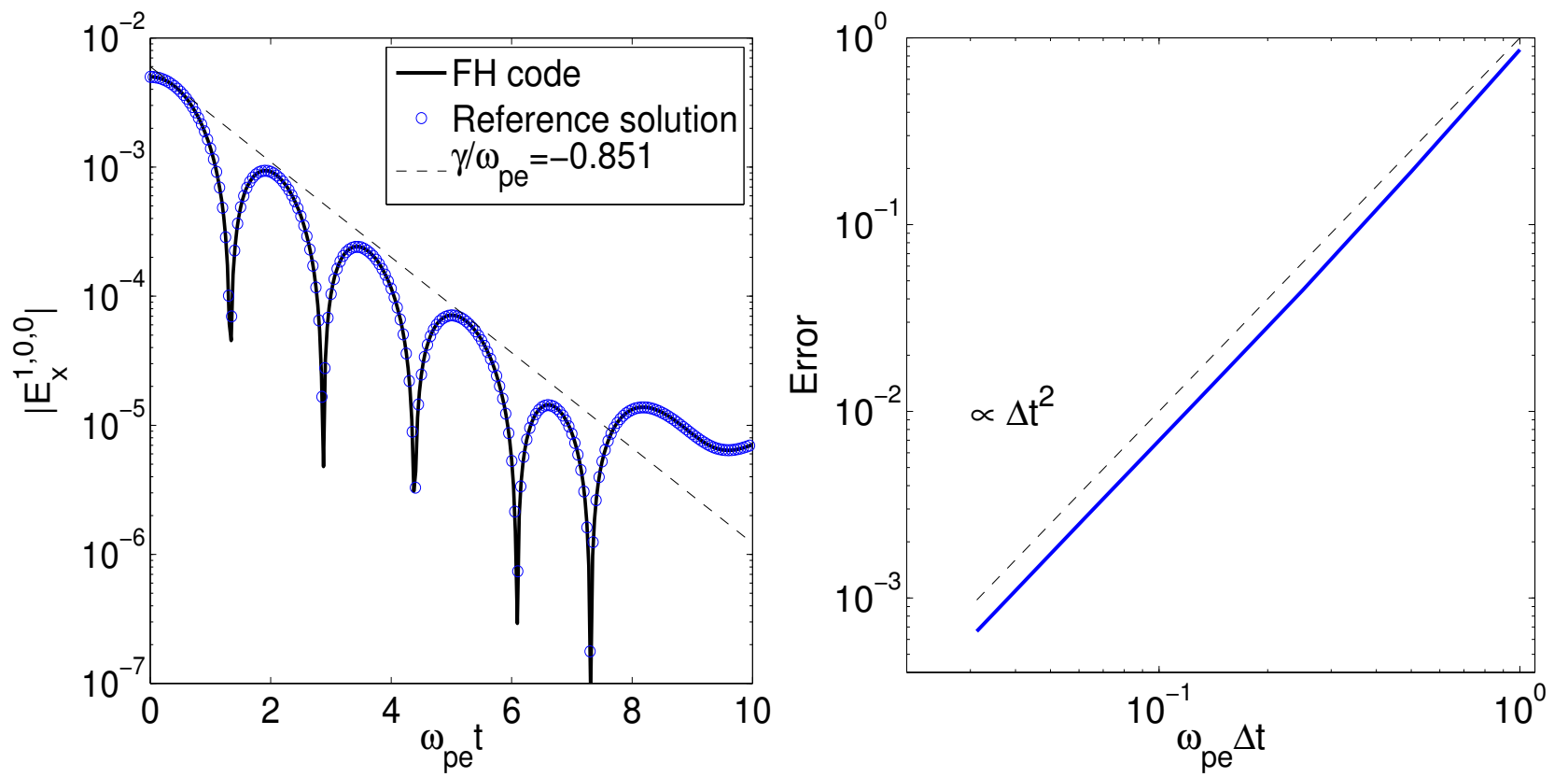

Fig. 2. Landau damping convergence study: (left) absolute value of the $x$-component of the electric field for the dominant mode $\left|E_{x}^{1,0,0}\right|$ versus time for the $\mathrm{FH}$ code (solid line, $\omega_{p e} \Delta t=0.3125$ ) and for the reference solution ('o'). The dashed line corresponds to the theoretical damping rate $\gamma / \omega_{p e}=-0.85$. (right) Error defined in Eq. (64) versus time step $\Delta t$. The dashed line corresponds to the theoretical second accuracy of the Crank-Nicolson scheme. 


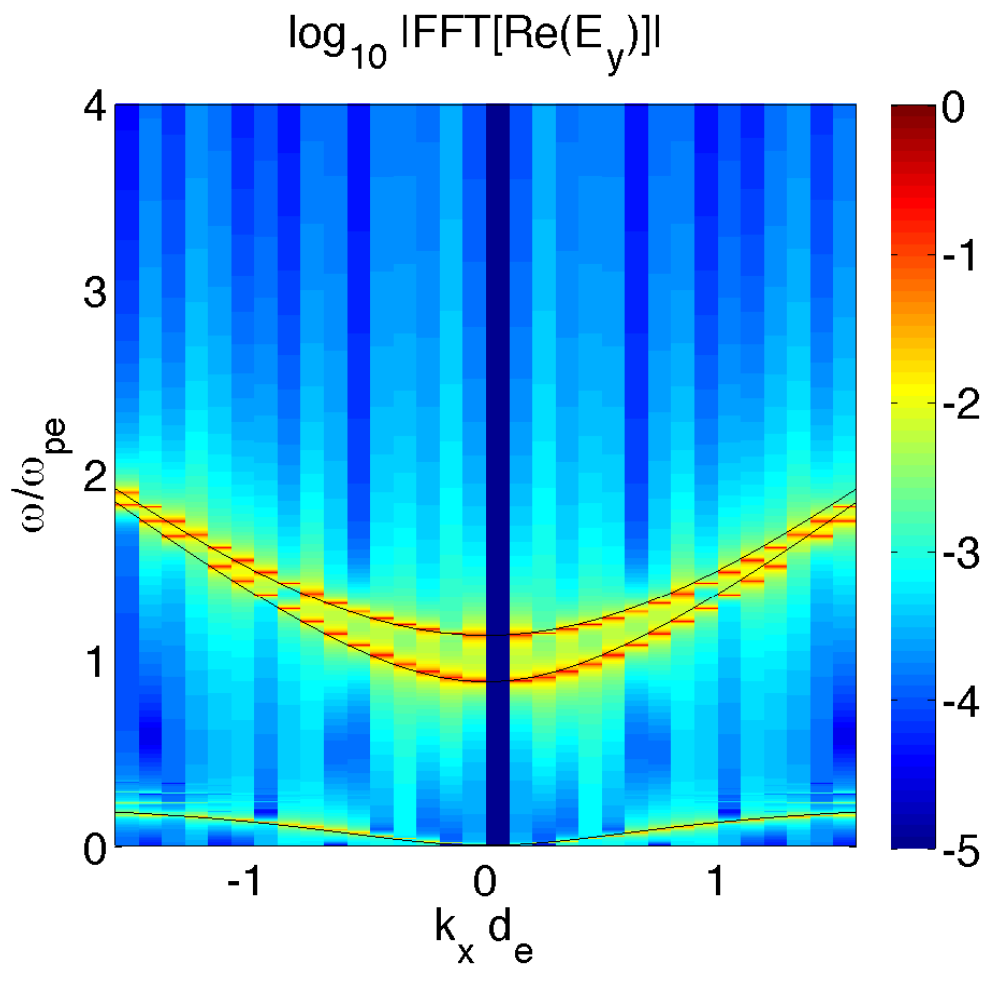

Fig. 3. High frequency waves propagating parallel to the background magnetic field $\mathbf{B}_{0}=(1,0,0)$ : wave spectrum $\log _{10}\left|\tilde{E}_{y}\left(k_{x}, \omega\right)\right|$ versus $k_{x}$ and $\omega$. The black solid lines are the dispersion curves obtained by Eq. (65) with $\theta=0$. 

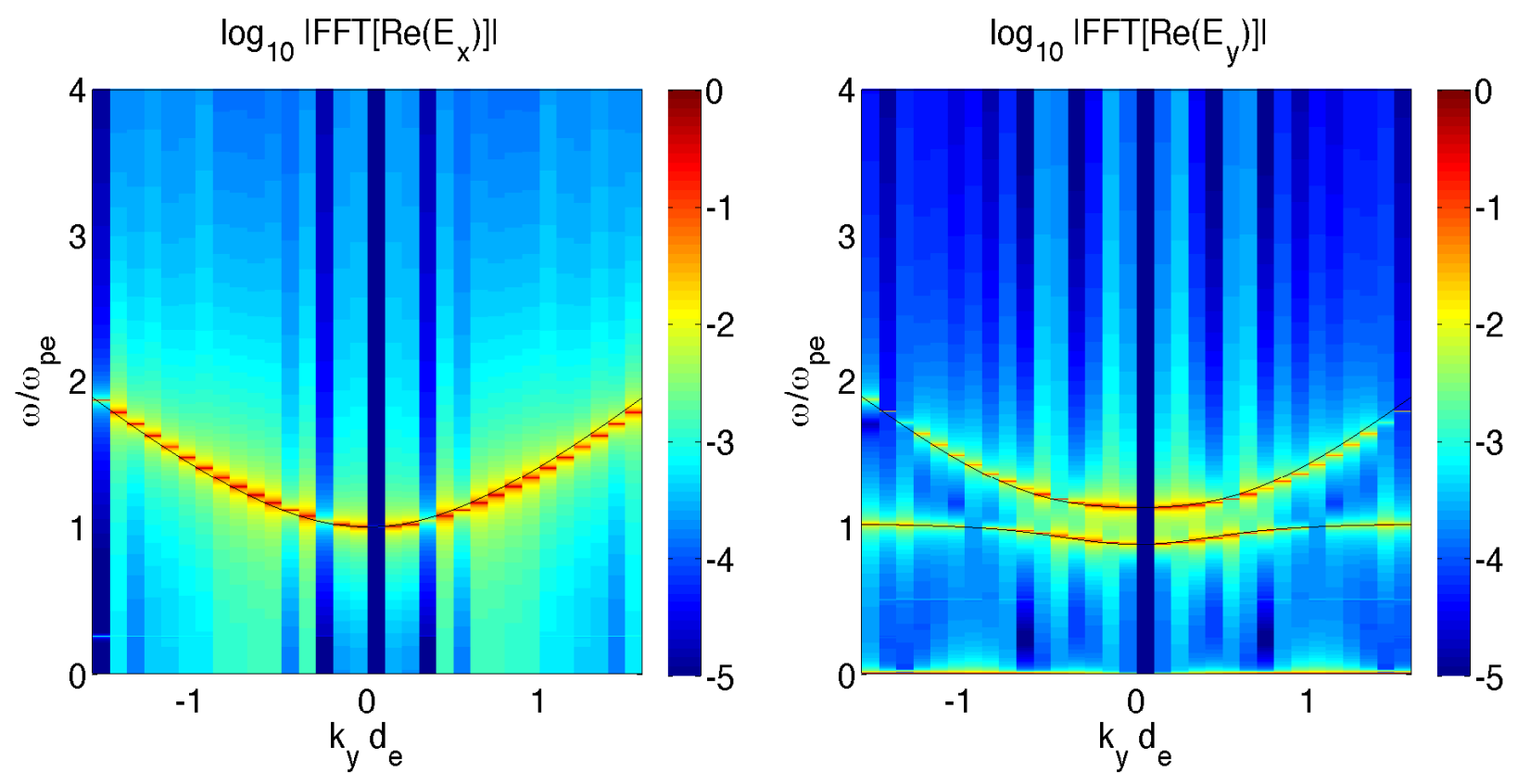

Fig. 4. High frequency waves propagating perpendicular to the background magnetic field $\mathbf{B}_{0}=(1,0,0)$ : wave spectrum $\log _{10}\left|\tilde{E}_{x}\left(k_{y}, \omega\right)\right|($ left $)$ and $\log _{10}\left|\tilde{E}_{y}\left(k_{y}, \omega\right)\right|$ versus $k_{y}$ and $\omega$. The black solid lines are the dispersion curves obtained by Eq. (65) with $\theta=\pi / 2$. 


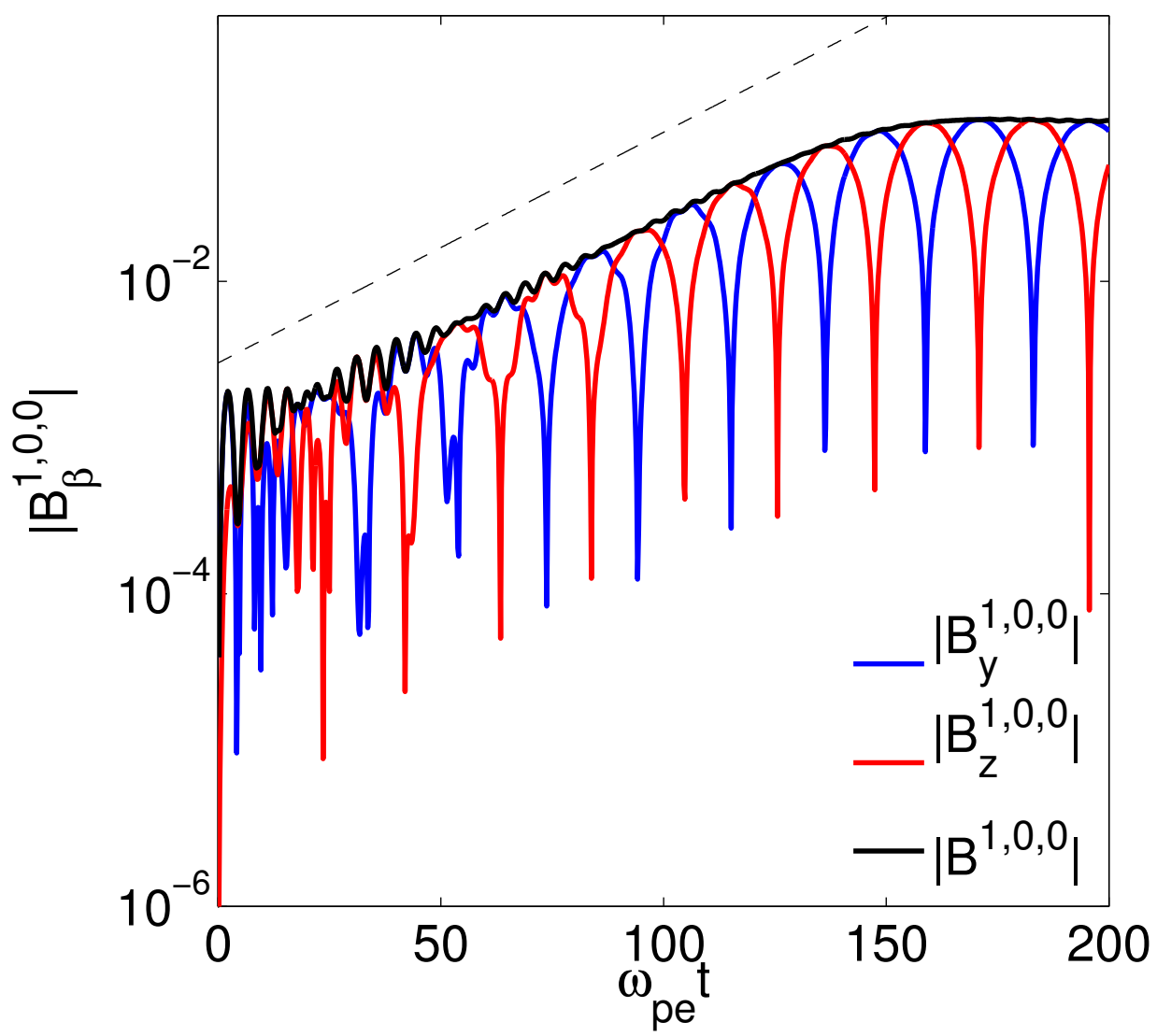

Fig. 5. One-dimensional whistler instability: time evolution of $\left|B_{y}^{1,0,0}\right|,\left|B_{z}^{1,0,0}\right|$ and $\left|B^{1,0,0}\right|=\sqrt{\left|\left(B_{x}^{1,0,0}\right)^{2}+\left(B_{y}^{1,0,0}\right)^{2}+\left(B_{z}^{1,0,0}\right)^{2}\right|}$ for $\Delta t=0.2$. The curve corresponding to the theoretical growth rate $\gamma_{\text {theory }} / \omega_{p e}=0.034$ is plotted with a dashed line. 


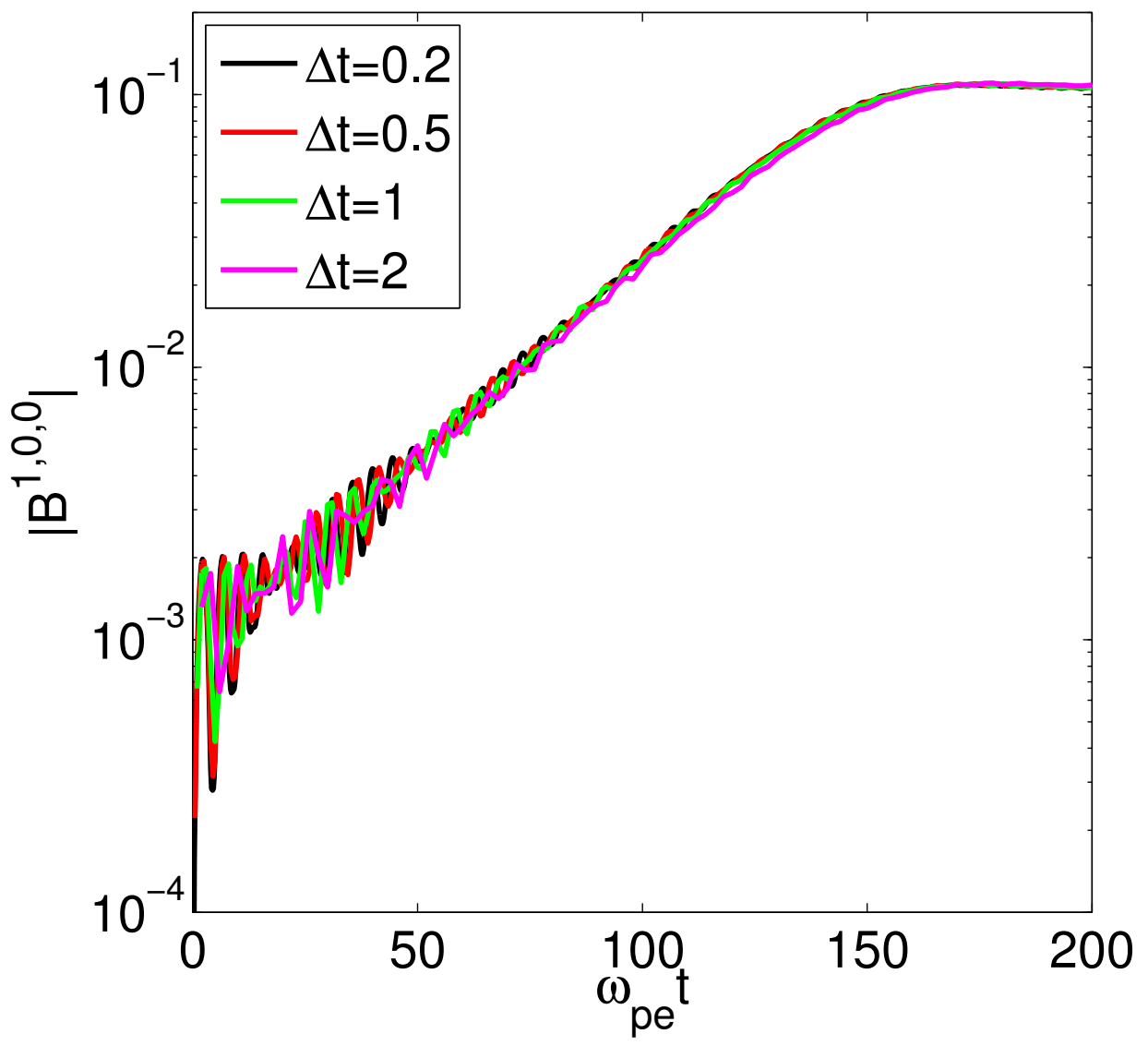

Fig. 6. One-dimensional whistler instability: time evolution of $\left|B^{1,0,0}\right|=\sqrt{\left|\left(B_{x}^{1,0,0}\right)^{2}+\left(B_{y}^{1,0,0}\right)^{2}+\left(B_{z}^{1,0,0}\right)^{2}\right|}$ changing $\Delta t$. 

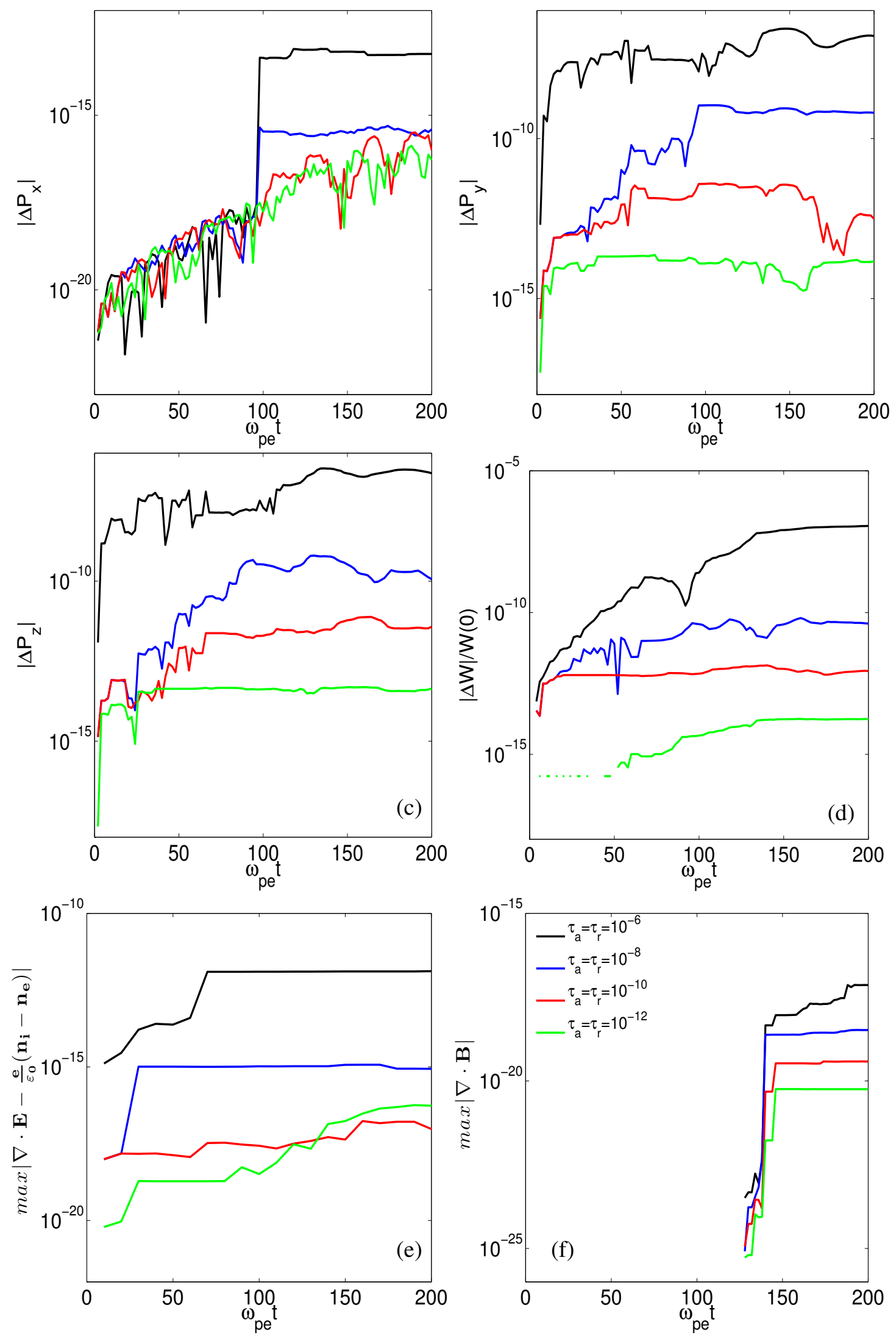

Fig. 7. One-dimensional whistler instability: numerical verification of the conservation laws derived in Sec. 3 (a)-(d), and of the constraints given by Gauss' law (4) (e) and $\nabla \cdot \mathbf{B}=0$ (f), varying the tolerance of the non-linear solver $\tau_{a}=\tau_{r}(\Delta t=2)$. In (d) and (f) some of the plot lines are missing where the specific quantity is exactly zero and not representable on the log scale of the plot. 

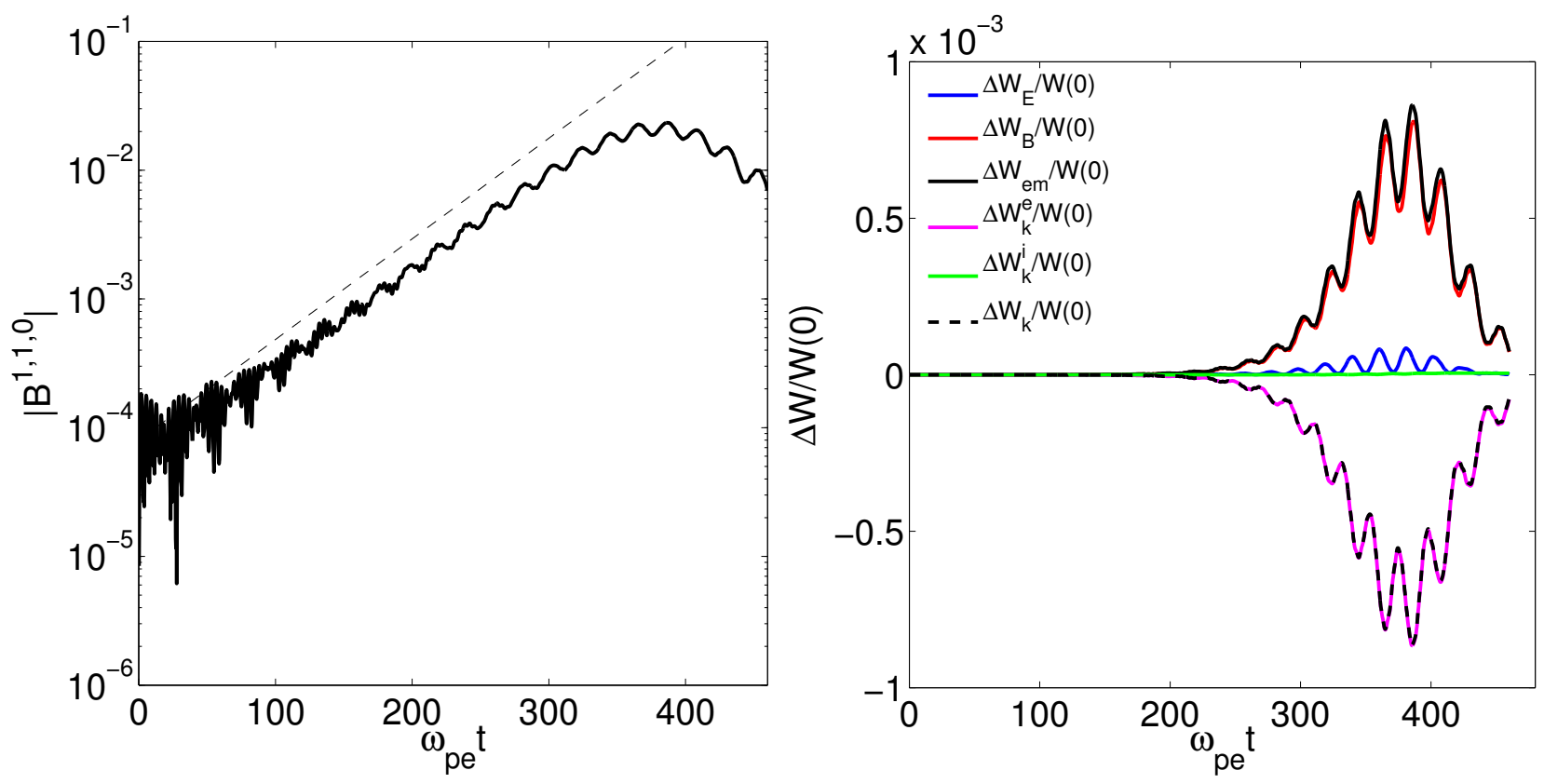

Fig. 8. Two-dimensional whistler instability: time evolution of $\left|B^{1,1,0}\right|=\sqrt{\left|\left(B_{x}^{1,1,0}\right)^{2}+\left(B_{y}^{1,1,0}\right)^{2}+\left(B_{z}^{1,1,0}\right)^{2}\right|}$ (left) and time variation of the relative change of various components of the energy, $\Delta W_{\alpha}=\frac{W_{\alpha}-W_{\alpha}(0)}{W(0)}$ (right). 


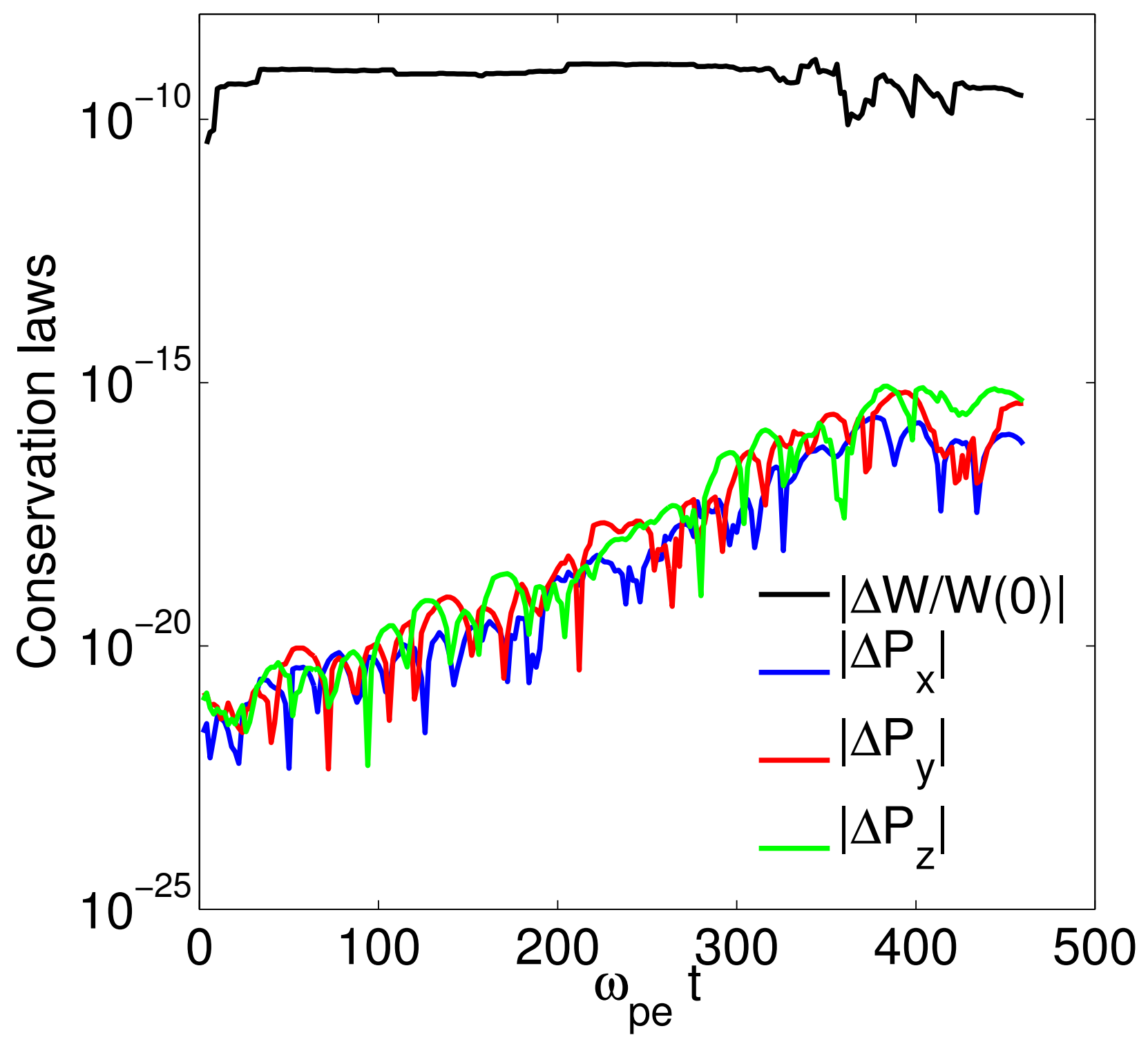

Fig. 9. Two-dimensional whistler instability: time evolution of $|\Delta W / W(0)|$ and $\left|\Delta P_{\beta}\right|$. 